\title{
Consistency of spherical, gravity-dominated dynamics with quasar high-ionization emission- line profiles
}

\section{Citation}

Kallman, T. R., B. J. Wilkes, J. H. Krolik, and Richard Green. 1993. "Consistency of Spherical, Gravity-Dominated Dynamics with Quasar High-Ionization Emission-Line Profiles." The Astrophysical Journal 403 (January): 45. doi:10.1086/172182.

\section{Published Version}

doi:10.1086/172182

\section{Permanent link}

http://nrs.harvard.edu/urn-3:HUL.InstRepos:30212150

\section{Terms of Use}

This article was downloaded from Harvard University's DASH repository, and is made available under the terms and conditions applicable to Other Posted Material, as set forth at http:// nrs.harvard.edu/urn-3:HUL.InstRepos:dash.current.terms-of-use\#LAA

\section{Share Your Story}

The Harvard community has made this article openly available.

Please share how this access benefits you. Submit a story.

\section{Accessibility}




\title{
CONSISTENCY OF SPHERICAL, GRAVITY-DOMINATED DYNAMICS WITH QUASAR HIGH-IONIZATION EMISSION-LINE PROFILES ${ }^{1}$
}

\author{
T. R. KaLlmaN \\ Laboratory for High Energy Astrophysics, Code 665, NASA/Goddard Space Flight Center, Greenbelt, MD 20771 \\ B. J. WILKES \\ Harvard/Smithsonian Center for Astrophysics, 60 Garden street, Cambridge, MA 02138 \\ J. H. KroLiK \\ Department of Physics and Astronomy, Johns Hopkins University, Baltimore, MD 21218 \\ AND \\ RICHARD GREEN \\ Kitt Peak National Observatory, National Optical Astronomy Observatories, ${ }^{2}$ \\ P.O. Box 26732, Tucson, AZ 85726 \\ Received 1991 October 14; accepted 1992 July 23
}

\begin{abstract}
Active galactic nucleus (AGN) emission-line profiles contain much information about the kinematics of the emission-line region. However, despite the large existing spectral data base, relatively little has been done to exploit this potential source of information fully because all existing analytic methods are strongly modeldependent and often involve large numbers of free parameters. In this paper we use line profile data to test a simple kinematic model - spherically symmetric gravitational free fall - in which we limit the number of free parameters by requiring physical self-consistency. The predictions of this model are fitted to high-resolution (1 $\AA$ ) spectra of the stronger rest-frame ultraviolet emission lines in 12 quasars with $z \simeq 2$. We find that if all the lines are radiated predominantly from the illuminated faces of the emission-line clouds, the profiles of Ly $\alpha$, $\mathrm{N}$ v $\lambda 1240$, and C IV $\lambda 1549$ can be simultaneously well fitted with very similar parameters for all 12 quasars. However, three points cast doubt on whether this model actually describes the broad emission line regions of these quasars: in some cases, there are no parameter choices which lead to acceptable fits if C III] $\lambda 1909$ is added to the list of predicted line profiles; the best-fit parameters found for the fits to $\operatorname{Ly} \alpha, \mathrm{N} v \lambda 1240$, and C IV $\lambda 1549$ are likely not to permit acceptable fits to other lines, once comparable profile data for them become available; finally, our results imply gravitational masses comparable to the most massive known galaxies, together with accretion rates which are likely to exceed known supply mechanisms. We therefore believe that spherically symmetric gravitational free fall does not correctly describe the dynamics of quasar broad emission line regions. Similar efforts applied to other dynamical scenario are likely to result in similarly severe constraints.
\end{abstract}

Subject headings: quasars: emission lines

\section{INTRODUCTION}

The optical and ultraviolet spectra of quasars are characterized by strong, broad emission lines covering a wide range of ionization. The apparent similarity of the profiles of these various lines in early, low-resolution spectra (Baldwin 1975) led to the suggestion that they originate in the same gas: the broad emission line region (BELR). Since then, much effort has been directed toward understanding the generation of these lines and the physical conditions in the BELR gas, leading to the development of the standard photoionization model.

The standard BELR model (as described, e.g., in Davidson $\&$ Netzer 1979) postulates that active galactic nucleus (AGN) emission lines arise in a large number of small clouds, all having very similar physical conditions, which surround a central compact source of continuum radiation. The heating and photoionization produced by this continuum is, in this model, the dominant mechanism contributing to line emission.

\footnotetext{
${ }^{1}$ Observations reported here were obtained at the Multiple Mirror Telescope Observatory, a joint facility of the Smithsonian Institution and the University of Arizona.

${ }^{2}$ Operated by the Association of Universities for Research in Astronomy, Inc., under cooperative agreement with the National Science Foundation.
}

This view has had significant success in accounting for several gross properties of AGN spectra, including the broad-line equivalent widths, the absence (or at least rarity) of hydrogen continuum absorption, and, most notably, the flux ratios of the UV emission lines. Problems of detail prevent complete acceptance of this model, however. For example, the $\mathrm{Ly} \alpha / \mathrm{H} \beta$ ratio, observed to be $\sim 5$ (Baldwin 1977), is a factor of several smaller than in any suggested model (Ferland \& Mushotzky 1982; Collin-Souffrin \& Dumont 1986; Kallman \& Krolik 1986), and the large (and uncertain) strength of the Fe II lines (Wills, Netzer, \& Wills 1985) has yet to find an adequate heat source. Most seriously, the quality of data available from recent variability studies (e.g., the NGC 5548 campaign: Clavel et al. 1991; Peterson et al. 1991; Krolik et al. 1991) has undercut the earlier satisfaction with single-zone models for the physical conditions in the BELR.

In fact, as a result of the recent variability campaigns, the first elements of a multizone picture of the BELR have begun to emerge. An essential part of that picture is the evidence that line-emitting material generally exists over a wide range of distances from the central object (see, for example, Krolik et al. 1991, Rees, Netzer, \& Ferland 1989, and Netzer 1987 for earlier papers discussing this possibility). 
Achieving a single-zone picture of the kinematics of the broad-line region is, in a sense, much easier than obtaining a single-zone picture of its physical conditions. We directly measure the emission-line profiles, and they can be immediately interpreted as the distribution function of line-of-sight velocities in that single zone. The counterpart of kinematics of the multizone picture obtained by variability studies is photoionization modeling of line profiles - that is, as a function of line-of-sight velocity, inferring the mean physical conditions via photoionization models. That is the goal of this paper.

Reaching this goal will be an important step toward determining the dynamics which create the large observed emissionline widths. If we could connect velocity directly with local ionizing flux and pressure, we would have very strong constraints on the form of the flow field and hence could distinguish rival dynamical models. Such tests would be especially valuable, for, despite 20 years of discussion, no consensus has yet formed around any dynamical models. It therefore seems at first sight surprising that this work should be new. The data required for it are much easier to acquire than the data required for variability studies (in fact, most of the spectra we use in this study date from the early 1980s). Yet most previous studies (e.g., Puetter \& Hubbard 1983; Kwan \& Carroll 1982; Ferland, Netzer, \& Shields 1979; Capriotti, Foltz, \& Byard 1979) were limited by restrictive assumptions (for example, that the clouds all have the same physical conditions) or by approximations in treating line emission processes (see, however, Rees et al. 1990; Mobasher \& Raine 1990). The unhappy fact which has retarded progress in this direction is that, unlike the isodelay surfaces onto which variability studies project the line emissivity, the isovelocity surfaces responsible for each segment of the line profiles are not known a priori. Consequently, any study in this area is deeply model-dependent.

The results obtained from profile studies to date have, in fact, been rather ambiguous. To first order the profiles are symmetric, arguing against dominant radial motions of optically thick clouds. Ly $\alpha$ production is particularly tightly constrained by this first fact (Wilkes \& Carswell 1982). However, the peak positions of the strongest ultraviolet lines (e.g. Ly $\alpha$, C IV 21549) are often blueshifted by $\sim 1000 \mathrm{~km} \mathrm{~s}^{-1}$ with respect to the quasar's systematic velocity (Gaskell 1982; Wilkes 1984, 1986; Espey et al. 1989). Such shifts can only be explained by radial motions. Clearly, more detailed analysis is required to make headway.

Our approach to this problem is to test individual dynamical models by comparing their predictions against high-quality line profiles from several quasars at $z \simeq 2$ observed at moderate spectral resolution. Because the predictions of any particular dynamical model require calculating line emission from a variety of different radii, it is vital to use a photoionization model which is as self-consistent as possible in making these predictions. In this paper we begin this program with what we consider to be the simplest dynamical scenario: that of radial gravitational free fall. The spirit of this work is to demonstrate the systematic behaviors of the synthetic profiles and to test for the qualitative success or failure of fits to observed profiles, rather than to derive the most accurate values for the various parameters involved. Indeed, as we will show, self-consistent profile modeling places such strong constraints on the parameters describing quasar BELR kinematics that it virtually rules out the simplest dynamical model, spherically symmetric gravitational free fall. In the following sections we describe first our photoionization modeling technique $(\S 2)$, then the sources of our observed profile data $(\S 3)$, detailed assumptions and techniques used to calculate model profiles $(\S 4)$, the results of our fits $(\S 5)$, and finally a discussion $(\S 6)$.

\section{BACKGROUND}

\subsection{The Standard BELR Model}

We assume that the broad emission lines are emitted from a large number of clouds surrounding a central compact object. The clouds are heated and ionized by the continuum radiation from the central object, and cool primarily via line emission. The lines are broadened by the cloud velocities, which can exceed $10^{4} \mathrm{~km} \mathrm{~s}^{-1}$. The arguments leading up to this scenario have been reviewed by Davidson \& Netzer (1979) and references therein. In the most general case the clouds may have a distribution of velocities and sizes at any point within the region, and the cloud number density distribution may have a complicated dependence on position within the region. For plausible cloud pressures and sizes the cloud free expansion time is short compared with the time associated with flow across the broad-line region, so there is likely to exist a heretofore unseen hot $\left(T \geq 10^{7} \mathrm{~K}\right)$ confining intercloud medium (ICM) whose heat source is not well understood (Mathews \& Ferland 1987). The ICM pressure, $P$, and the ionizing radiation flux from the central object, $F_{c}$, are unique functions of position, and hence so is the "ionization parameter" which determines, crudely, the cloud emission properties. We adopt the definition $\Xi=F_{c} /(c P)$ (Krolik, McKee, \& Tarter 1981), although several other equivalent definitions are applicable.

In the most general model for the BELR the number of applicable free parameters is forbiddingly large. In order to make progress in modeling it is necessary to reduce the complexity of the model for cloud velocities and number density. In this spirit we will assume that the cloud velocities are a unique function of position, that they are purely radial, and that the cloud number density distribution and velocity depend only on radial distance from the continuum source. Comparable simplification can be achieved by other sets of assumption, e.g., that the cloud motions are purely orbital. We have chosen these in order to explore their consequences for the study of line profiles, not because we consider them to be necessarily more correct than any others.

\subsection{Line Profile Basics}

Under the assumptions of the standard model, described above, the profile of a line $l$ with rest energy $\varepsilon_{0}$ observed at an energy $\varepsilon$ is given by

$$
\begin{array}{r}
F_{\varepsilon}=\frac{1}{4 \pi D_{L}^{2}} \int d^{3} r n_{\mathrm{cl}}(\boldsymbol{r}) A_{\mathrm{cl}}(\boldsymbol{r}) F_{l}\left[F_{c}, P(\boldsymbol{r})\right] e^{-\tau(\boldsymbol{r})} R\left[\theta ; F_{c}, P(\boldsymbol{r})\right] \\
\times \delta\left[\varepsilon(1+z)-\varepsilon_{0}\left(1-\frac{v \cos \theta}{c}\right)\right],
\end{array}
$$

where $D_{L}$ is the luminosity distance to the quasar; $n_{\mathrm{cl}}$ is the local density of emitting clouds; $A_{\mathrm{cl}}$ is the mean projected area of each cloud; $F_{l}$ is the surface brightness is line $l$ from each cloud and is a function of the local ionizing continuum flux $F_{c}$; $P$ is the gas pressure; $\tau(\boldsymbol{r})$ is the absorption optical depth from the observer to the emitting region; $v$ is the cloud velocity; and $R$ is the relative amount of the line emission radiated into angle $\theta$, the angle between the line of sight and the line connecting the continuum source with the position of the emitting material. It is normalized such that $\int d \Omega R=4 \pi$. The $\delta$ - 
function contains the Doppler constraint. Equation (1) also incorporates the assumption that the cloud velocities are radial relative to the continuum source, and that the velocities are a unique of position, i.e., that turbulence is unimportant.

If the clouds are spherically distributed, equation (1) may be simplified to a single integral:

$$
F_{\varepsilon}=\frac{c(1+z)}{2 \varepsilon_{0} D_{L}^{2}} \int r^{2} d r \frac{d C}{d r} \frac{F_{l}\left[F_{c}, P(r)\right]}{v(r)} e^{-\tau(r)} R\left[\theta ; F_{c}, P(r)\right],
$$

where $\varepsilon_{0}, c$, and $v(r)$ in the denominator come from integrating over the $\delta$-function, $\theta=\cos ^{-1}\left[c / v(r)\left(\varepsilon / \varepsilon_{0}-1\right)\right]$ is the angle between the observer's line of sight and the radius vector from the central source, and the covering factor is defined as

$$
C(r)=\int_{r}^{\infty} d r^{\prime} n_{\mathrm{cl}}\left(r^{\prime}\right) A_{\mathrm{cl}}\left(r^{\prime}\right)
$$

\subsection{Cloud Conditions}

The quantities which characterize the cloud line radiation under the standard model are the cloud gas pressure $P$, the ionization parameter $\Xi$, the column density $N$, the element abundances, and the spectrum of the ionizing continuum. The latter three are not prescribed by the dynamical assumptions described in the previous section, and will be discussed in $\$ 4$.

The gas pressure is determined by the temperature and dynamics of the BELR gas. The hot phase (ICM) will almost certainly dominate the mass budget of the BELR gas, and we adopt the assumptions that the ICM velocity distribution is the same as that of the clouds, and that there are no sources or sinks of ICM gas in the BELR. Owing to its relatively low density $\left(\leq 10^{7} \mathrm{~cm}^{-3}\right)$, the ICM temperature will be determined jointly by the "Compton temperature," which is determined by the shape of the continuum spectrum and whose value lies in the range $T_{\text {hot }} \simeq 10^{7}-10^{8} \mathrm{~K}$ (e.g., Krolik et al. 1981; Kallman \& Mushotzky 1986) and by other heating processes. The ionization parameter and pressure are then given by

$$
\begin{aligned}
\Xi(r) & =\frac{L \mu m_{\mathrm{H}} v(r)}{\dot{M} k T_{\mathrm{hot}}(r) c}, \\
P(r) & =\frac{\dot{M} k T_{\text {hot }}(r)}{4 \pi r^{2} v(r) \mu m_{\mathrm{H}}},
\end{aligned}
$$

where $\dot{M} /\left(4 \pi r^{2}\right)$ is the hot phase mass flux and $\mu \mathrm{m}_{\mathrm{H}}$ is the mean molecular weight.

\subsection{Photoionization Models}

There is general agreement on the line fluxes emitted by BELR clouds under the standard model assumptions among various authors such as Kwan \& Krolik (1981), Ferland \& Netzer (1983), Weisheit, Shields, \& Tarter (1982), and Krolik \& Kallman (1988). Although the results of such models are complicated in detail, some generalizations may be made concerning the behavior of line fluxes.

In this paper we are concerned primarily with understanding the behavior of lines whose rest wavelengths are in the UV, and whose strengths are relatively large; these include hydrogen Ly $\alpha, C$ Iv $\lambda 1549, N$ v $\lambda 1240, C$ III] $\lambda 1909$, and O vi $\lambda 1034$. The strongest of these, Ly $\alpha$ and C IV $\lambda 1549$, are the dominant coolants throughout a large fraction of the volume of any given cloud, and so tend to be approximate "bolometers" of the cloud heating. Here and in what follows we will refer to a "bolometric" line as one whose emitted flux is directly pro- portional to the ionizing continuum flux incident of the cloud $\left(F_{c}\right.$ in eq. [1]). For these lines $F_{l} \propto P \Xi$. However, we stress that this behavior is only a crude approximation to the true dependence of these lines on the cloud conditions, and that in our detailed models (presented in $\S 5$ ) we treat these lines as accurately as possible and so do not depend on the bolometric assumption.

The lines of $\mathrm{N} v \lambda 1240$ and $\mathrm{O}$ vI $\lambda 1034$ arise in the most highly ionized part of the cloud, where these ions are still trace species. As a result the line strengths can depend very sensitively on ionization parameter, typically $F_{l} \sim \Xi^{\beta}$, where $\beta=1-3$. The C III] $\lambda 1909$ line arises from less highly ionized gas than the other lines in this study, and is strongly affected by collisional suppression. Thus it has a weaker dependence on ionization parameter and pressure than the other lines for plausible quasar conditions.

It is important to point out that most of the photoionization model calculations which have been carried out, including ours, rely on the escape probability assumption in order to treat the transfer of cooling radiation out of the clouds. Although this assumption is the only one which is currently computationally feasible for a project such as ours, it is likely to be incorrect at some level when the cloud column density is very large, owing to interlocking between lines and continuum, partial redistribution in the line scattering, or whenever continuous opacity is an important line photon destruction mechanism.

\subsection{Cloud Radiation Pattern}

Because the shape of the line profile depends both on the angular radiation pattern of the line and on the geometry of emitting material, we must use our knowledge of line radiation transfer to separate the two effects. This is complicated by uncertainties concerning the cloud shapes, and by the current primitive understanding of the "limb-darkening" behavior of even the simplest of standard clouds. In particular, there exists no self-consistent treatment of the transfer of radiation within these clouds which includes both the angular dependence of the radiation field and an accurate treatment of the thermal balance.

In spite of these uncertainties, we can make certain educated guesses about the cloud angular radiation pattern based on the standard model with clouds of column density $\sim 10^{23} \mathrm{~cm}^{-2}$. Of all the lines we wish to model, only C III] $\lambda 1909$ is an intercombination line whose oscillator strength is very small and so is likely to escape the clouds isotropically because of the small optical depth it encounters. In the case of $N v \lambda 1240$ the optical depth is likely to be large, but it is probably not as large as the thermalization depth, i.e., the optical depth such that the collisional de-excitation rate matches the spontaneous emission rate times the escape probability. Its radiation pattern then depends strongly on the shape of the emitting cloud. C IV $\lambda 1549$ is almost in this category, but its optical depth can, in some cases, be large enough to begin thermalization. When that happens, its angular radiation pattern favors the illuminated side. Ly $\alpha$ is likely to present the most extreme case of expected anisotropy. If the Lyman edge optical depth is $\leq O(10)$, its angular radiation pattern will be similar to the resonance lines such as $\mathrm{C}$ IV $\lambda 1549$ and $\mathrm{N} v \lambda 1240$. However, if low-ionization lines such as $\mathrm{Mg}$ II $\lambda 2800$ are produced from the same material, and if collisional enhancement of the Balmer lines is required to match the observed line ratios (as is often the case), the Lyman edge optical depth must be much greater 
than this, and Ly $\alpha$ will be thermalized. In that case, the illuminated side is very strongly favored: its surface brightness in Ly $\alpha$ can be easily two or three orders of magnitude greater than that of the shadowed side (Kwan \& Krolik 1981; Ferland et al. 1979).

One important consequence is that it is difficult to account for spectra in which the C IV asymmetry exceeds that of Ly $\alpha$ with cloud models whose column density is $\sim 10^{23} \mathrm{~cm}^{-2}$. As we will show in the next section, $\mathrm{C}$ IV is frequently more asymmetric than Ly $\alpha$ in our quasar sample. This can be explained if the cloud column densities are $\geq 10^{25} \mathrm{~cm}^{-2}$, greatly exceeding the standard value of $\sim 10^{23} \mathrm{~cm}^{-2}$. The cloud is then opaque to all line photons owing to various opacity sources, including continuous absorption by the hydrogen Balmer continuum, dust, line-blanketing effects by both neutral atoms and molecules, and free-free absorption. Little theoretical modeling of these processes in quasar clouds has been carried out (see, however, Ferland \& Persson 1989; Kallman \& Lepp 1987). Therefore, at high column densities, we expect the clouds to radiate with comparable asymmetries for all lines.

\subsection{Intercloud Absorption}

Line strengths and asymmetries may also be affected by absorption or scattering by material between the clouds (the intercloud medium). This material may be free electrons (Kallman \& Krolik 1986; Shields \& McKee 1981), dust, or perhaps a dense ensemble of optically thick clouds or cloudlike material such as an accretion disk. If so, the profile shape and asymmetry will be affected by the geometrical distribution and optical depth of the absorbers, neither of which is constrained by standard BELR models. If the absorber distribution is centered on the compact object, intercloud absorption preferentially suppresses the lines emitted by clouds on the far side of the central object relative to the observer. On the other hand, asymmetries intrinsic to the clouds suppress lines from the near side of the central object. The observed line asymmetries (i.e., red or blue) depend on which mechanism is acting (or dominating) and the direction of the cloud motion relative to the central object (i.e., accretion or outflow).

\subsection{Analytic Profiles}

The physical quantities which a complete dynamical model provides include the run of cloud velocity with radius, $v(r)$, and the run of hot phase temperature, $T_{\text {hot }}(r)$. Here and in what follows we will make use of the fact that, for the range of ICM temperatures consistent with Compton heating models (e.g., Krolik et al. 1981), $T_{\text {hot }} \leq 10^{8} \mathrm{~K}$, the mass budget in the BELR gas flow is dominated by the hot phase gas. We will assume that the hot phase mass flux in the BELR is conserved, i.e., that the sources and sinks of the gas are outside the region. We also assume that the cloud velocity and hot phase temperature are described by power laws, i.e., $v(r)=v_{0}\left(r / r_{0}\right)^{\alpha}$ and $T_{\text {hot }}=$ $T_{0}\left(r / r_{0}\right)^{\delta}$, where $v_{0}, T_{0}$, and $r_{0}$ are suitably chosen fiducial values. These assumptions are motivated by the desire for simplicity and by dynamical calculations of the infalling BELR material such as those of Krolik \& London (1983), which show the approximate validity of the power-law parameterization. The variation in hot phase temperature with radius is due to the fact that the Compton heating and cooling time scales can exceed the flow time scale, so that material flowing into the BELR can retain a memory of its initial temperature. We do not make any assumption concerning the sources or sinks of cloud material; that is motivated by the variety of processes by which clouds can be created or destroyed (e.g., Mathews 1986; Mathews \& Ferland 1987; Krolik 1988) and by the fact that cloud creation and destruction probably provide only a perturbation to the mass and energy budget of the gas flow through the BELR. Referring to equations (4) and (5), the cloud ionization parameter and pressure are then given by

$$
\begin{gathered}
\Xi(r)=\frac{\eta c \mu m_{\mathrm{H}} v_{0}}{k T_{0}}\left(\frac{r}{r_{0}}\right)^{\alpha-\delta}, \\
P(r)=\frac{L_{c} k T_{0}}{4 \pi \eta c^{2} r_{0}^{2} v_{0} \mu m_{\mathrm{H}}}\left(\frac{r}{r_{0}}\right)^{\delta-\alpha-2},
\end{gathered}
$$

where $\eta=L_{c} /\left(\dot{M} c^{2}\right)$ is the accretion efficiency if the BELR flow rate is compared with that required to power the continuum from the compact object.

With such a power-law distribution of dynamical quantities, it is straightforward to construct analytic estimates for line profiles. In order to do so, we assume for the purpose of illustration that the line fluxes emitted by clouds also follow a power law in pressure and ionization parameter, i.e.,

$$
F_{l}=F_{0}\left(\frac{\Xi}{\Xi_{0}}\right)^{\beta}\left(\frac{P}{P_{0}}\right)^{\gamma} \text {. }
$$

This parameterization is only a crude approximation to the actual line fluxes emitted by the clouds; a more accurate treatment is used in the models presented in $\S 4$. Throughout the rest of this paper we assume that the covering fraction follows a similar functional form,

$$
\frac{d C}{d r}=\frac{C_{0}}{r_{0}}\left(\frac{r}{r_{0}}\right)^{\epsilon}
$$

If so, it is straightforward to show that the profile is given by

$$
F_{\varepsilon}=\frac{r_{0}^{2}}{D^{2}} \frac{F_{0} C_{0}}{2 \varepsilon_{0}(1+\zeta)} \frac{c}{v_{0}}\left(\frac{v_{\max }}{v_{0}}\right)^{1+\zeta}\left\{1-\left[\frac{\max \left(c x, v_{\min }\right)}{v_{\max }}\right]^{1+\zeta}\right\}
$$

for $\zeta \neq 1$, and

$$
F_{\varepsilon}=\frac{r_{0}^{2}}{D^{2}} \frac{F_{0} C_{0}}{2 \varepsilon_{0}} \frac{c}{v_{0}} \ln \left[\frac{\max \left(c x, v_{\min }\right)}{v_{\max }}\right]
$$

for $\zeta=1$, where

$$
x=\left(\frac{\varepsilon}{\varepsilon_{0}}-1\right) \frac{v_{\max }}{c}
$$

and

$$
1+\zeta=[(\alpha-\delta)(\beta-\gamma)-2 \gamma+3+\epsilon-\alpha] \alpha .
$$

Equation (10) shows that the shape of the profile depends on the exponent $\zeta$ in the sense that the concavity of each half of the profile (between line center and the extreme line wing) depends on the sign of $1+\zeta$. For $1+\zeta<0$ the halves are "concave up," that is, the absolute value of their slope reaches a maximum at line center, and for $1+\zeta<0$ the converse is true. For bolometric lines as described in the previous subsection, i.e., $\beta=\gamma=1$, this behavior depends only on the cloud covering fraction distribution, i.e., $1+\zeta=\alpha(1-\alpha+\epsilon)$. So $\epsilon=0$, corresponding to a covering fraction which is independent of velocity, produces logarithmic profiles, while $\epsilon=1$ / $\alpha+\alpha-1$ produces triangular profiles. More negative values of $\epsilon$, corresponding to greater contributions to the covering 
fraction at lower velocities, produce profiles which have downward concavity, and vice versa. For nonbolometric lines we can define $\zeta_{\text {crit }}=(\alpha-\delta)(\beta-\gamma)-2 \gamma+2$, so that $1+\zeta=$ $-\left(\zeta_{\text {crit }}+\epsilon\right) / \alpha+1$. As will be shown in the next section, these results are not changed qualitatively when the results of real photoionization models are used to calculate the line fluxes emitted by the clouds.

Based on the discussion so far, we can describe qualitatively the dependence of the line profile on the various free parameters involved. The accretion efficiency $\eta$ affects the ionization parameter and pressure scales and hence the relative strengths of the various lines with differing values of $\beta$ and $\gamma$. The ionizing luminosity, $L_{c}$, is constrained by observations, although uncertainties about the shape of the spectrum and the distance allow for some freedom in choosing this quantity; differing values will affect the pressure scale. The velocity scale, $v_{0}$, affects line widths and the run of ionization parameter and pressure. The minimum and maximum cloud velocities affect the "peakiness" of the line; at wavelengths $\left|\varepsilon / \varepsilon_{0}-1\right| \leq v_{\min } / c$ the line profile will be flat (cf. eq. [10]). The hot phase temperature fiducial value, $T_{0}$, effects both the ionization parameter and the pressure, but in the opposite sense. In addition, lower hot phase temperatures are more likely to imply intercloud absorption or scattering. The radial dependence of $T_{\text {hot }}$, $\delta=d \log T_{\text {hot }} / d \log r$, also affects the ionization parameter and presssure distributions; if $\delta=\alpha$, the ionization parameter is independent of radius. The covering fraction affects line shapes and equivalent widths in the manner already described. The line strengths are also affected by the photoionization model parameters: ionizing spectrum, cloud column, and element abundances. These will be discussed further in $\S 4$. The cloud radiation pattern and the intercloud absorption both affect the line asymmetries and may also affect the line strengths in situations where two lines are blended together.

\section{THE QUASARS}

The quasar sample is a heterogeneous collection of 12 bright, high-redshift $(z \sim 2)$ quasars for which high signal-to- noise, moderate-resolution $(\sim 1 \AA)$ optical spectral data are available. Their vital statistics are given in Table 1 , along with reference to previous publication of the spectra (if any). Observational details for previously unpublished data are given in Table 2.

All spectra were reduced in the standard manner and rebinned onto a wavelength scale, preserving as closely as possible the original data sampling. Flux calibration onto a relative scale was performed with reference to a standard star observed on the same night with the identical spectrograph setup. The absolute spectrophotometric accuracy is poor owing to the use of small apertures $\left(\sim 1^{\prime \prime}-2^{\prime \prime}\right)$, but the relative accuracy across an individual emission-line profile is generally within the noise level of the spectrum.

For comparison with model profile predictions, the spectra were processed in the following manner:

1. First, absorption lines were removed from the vicinity of the emission-line profiles by fitting a sum of Gaussian profiles to each absorption feature/blend as appropriate and subtracting the fit from the data. Figure 1 shows the original spectra, on a correctly normalized flux scale (see below).

2. A polynomial fit was made to the continuum over predefined wavelength regions, in the quasar rest frame, known to be uncontaminated by emission lines (Table 3), using the redshift given in Table 1. The redshift used in this process for each quasar is given in Table 1. Fits were inspected by eye and modified if appropriate.

3. The best-fit continuum was subtracted from the spectrum to isolate the line profiles.

4. An error range of $\pm 1 \sigma$ around the continuum fit was used as the tolerance for the profile model fitting. The value of this error is dominated by the ends of the spectrum, where the signal-to-noise is lowest. It is generally $\sim 3 \sigma$ over most of the spectrum.

Since the absolute flux level from the spectral data is inaccurate, the continuum level of each quasar was normalized so that the level at $4400 \AA$ (5500 $\AA$ for $V$-magnitudes) agrees with

TABLE 1

Quasars Included in This Study

\begin{tabular}{|c|c|c|c|c|c|c|c|}
\hline Object & R.A. & Decl. & $z_{\text {BLR }^{a}}{ }^{\mathbf{a}}$ & $z_{\mathrm{OI}}{ }^{b}$ & $B^{\mathrm{c}}$ & $B$ Reference & $\begin{array}{l}\text { Spectrum } \\
\text { Reference }\end{array}$ \\
\hline Q0000-398 & $00^{\mathrm{h}} 00^{\mathrm{m}} 30.3$ & $-39^{\circ} 48^{\prime} 57^{\prime \prime}$ & 2.830 & 2.841 & $18.8^{\mathrm{d}}$ & OS80 & W86 \\
\hline Q0207-398 & $0207 \quad 24.3$ & -395350 & 2.813 & 2.821 & 17.35 & A85 & W86 \\
\hline PKS $0304-392$ & 030418.8 & -391734 & 1.965 & 1.979 & $17.6^{\mathrm{d}}$ & OS 80 & W86 \\
\hline Q1101-264 $\ldots \ldots \ldots \ldots \ldots$ & 110100.0 & -262908 & 2.144 & $\cdots$ & 16.08 & A85 & W86 \\
\hline Ton $1530 \ldots \ldots \ldots \ldots \ldots \ldots$ & 122256.6 & +225149 & 2.046 & 2.056 & 15.49 & SG83 & \\
\hline PG $1247+265 \ldots \ldots \ldots \ldots \ldots$ & 124739.0 & +264728 & 2.038 & 2.047 & 15.53 & SG83 & \\
\hline PKS $1331+170^{\mathrm{e}} \ldots \ldots \ldots \ldots$ & $1331 \quad 10.0$ & +170420 & 2.077 & 2.090 & 16.84 & A85 & W86 \\
\hline Mrk $679 \ldots \ldots \ldots \ldots \ldots \ldots$ & $1421 \quad 17.5$ & +330555.5 & 1.904 & 1.912 & 16.97 & A85 & \\
\hline $1559+089^{\mathrm{f}} \ldots \ldots \ldots \ldots \ldots \ldots$ & 155957.8 & +085353 & 2.269 & 2.273 & $16.7^{\mathrm{g}}$ & $\mathbf{H}$ & W86 \\
\hline PKS $2044-168 \ldots \ldots \ldots \ldots$ & 204431.0 & -165012 & 1.937 & 1.942 & 17.55 & A85 & W86 \\
\hline Q22204-408 .............. & 220433.1 & -405139 & 3.170 & 3.182 & $17.57^{\mathrm{h}}$ & A85 & W86 \\
\hline PKS $2212-299 \ldots \ldots \ldots \ldots$ & 221225 & -295920 & 2.700 & 2.708 & 17.58 & A85 & W86 \\
\hline \multicolumn{8}{|c|}{ a Mean redshift derived from broad lines. } \\
\hline \multicolumn{8}{|c|}{ b Redshift derived from $O_{\mathrm{I}} \lambda 1304$ (Wilkes 1984,1986 ). } \\
\hline \multicolumn{8}{|c|}{ c Observed $B$-magnitude (unless otherwise noted). } \\
\hline \multicolumn{8}{|c|}{ d Continuum magnitude at $1475 \AA$ in the quasar rest frame. } \\
\hline \multicolumn{8}{|c|}{ e Spectrum presented here is from Table 1 of the 1983 data. } \\
\hline \multicolumn{8}{|c|}{ f Spectrum presented here is a sum of 1983 Table 2 data and the earlier data. } \\
\hline \multirow{2}{*}{\multicolumn{8}{|c|}{$\begin{array}{l}B_{\mathrm{J}}=16.6 \pm 0.1 \text {. The } B \text {-magnitude was determined using the conversion of Blair \& Gilmore } 1982 \text { with } B-V=0.3 \text {, an } \\
\text { erage color for a } z=2 \text { quasar in Hewitt \& Burbidge } 1989 \text {. } \\
{ }^{\mathrm{g}} V \text {-magnitude. }\end{array}$}} \\
\hline & & & & & & & \\
\hline \multicolumn{8}{|c|}{ REFERENCES.-A85: Adam 1985; H: C. Hazard, private communication; OS80: Osmer \& Smith 1980; SG83: Schmidt \& } \\
\hline
\end{tabular}


TABLE 2

Details of New Observations

\begin{tabular}{|c|c|c|c|c|c|}
\hline Quasar & $\begin{array}{l}\text { Date } \\
\text { (UT) }\end{array}$ & $\begin{array}{l}\text { Telescope } \\
\text { and } \\
\text { Instrument }\end{array}$ & $\begin{array}{l}\text { Time } \\
(\mathrm{s})\end{array}$ & $\begin{array}{c}\text { Resolution } \\
(\AA)\end{array}$ & $\begin{array}{c}\text { Range of } \lambda \\
(\AA)\end{array}$ \\
\hline \multirow[t]{2}{*}{ Ton $1530 \ldots \ldots \ldots \ldots \ldots$} & 1984 Jan 29 & MMT & 4000 & 1 & $3330-4230$ \\
\hline & & Blue spectrograph & 2000 & $\ldots$ & $4100-4950$ \\
\hline \multirow[t]{2}{*}{ PG $1247+265 \ldots \ldots \ldots \ldots \ldots$} & 1983 May 17 & MMT & 7000 & 1 & $3500-4400$ \\
\hline & & Blue spectrograph & 2600 & 1 & $4200-5100$ \\
\hline \multirow[t]{2}{*}{ PKS $1331+170 \ldots \ldots \ldots \ldots$} & 1983 Apr 17 & SO $90^{\prime \prime}$ & 3600 & 1 & $3200-4300$ \\
\hline & & B\&C spectrograph ${ }^{\mathbf{a}}$ & 6000 & 2 & $4200-5800$ \\
\hline \multirow[t]{2}{*}{ Mrk $679 \ldots \ldots \ldots \ldots \ldots \ldots$} & 1983 May 17 & MMT & 7100 & 1 & $3350-4250$ \\
\hline & & Blue spectrograph & 2600 & $\ldots$ & $4100-5000$ \\
\hline \multirow[t]{2}{*}{$1559+089$} & 1983 Apr 17 & SO $90^{\prime \prime}$ & 8400 & 2 & $4200-5800$ \\
\hline & & B\&C spectrograph ${ }^{a}$ & $\ldots$ & $\ldots$ & $\ldots$ \\
\hline
\end{tabular}

a Boller \& Chivens spectrograph mounted on the Steward Observatory 90 inch (2.3 m) telescope.

TABLE 3

Emission-Line-Free ContinuUm Regions Used for Polynomial Fit

\begin{tabular}{cc|cc}
\hline \hline $\begin{array}{c}\text { Lower Bound } \\
(\AA)\end{array}$ & $\begin{array}{c}\text { Upper Bound } \\
(\AA)\end{array}$ & $\begin{array}{c}\text { Lower Bound } \\
(\AA)\end{array}$ & $\begin{array}{c}\text { Upper Bound } \\
(\AA)\end{array}$ \\
\hline 1040 & 1180 & 1430 & 1505 \\
1280 & 1300 & 1675 & 1860 \\
1315 & 1330 & 1950 & $2160^{\mathrm{a}}$ \\
1340 & 1380 & & \\
\hline
\end{tabular}

a This band includes possible Fe II emission at $2160 \AA$, but the two quasars where this emission could be seen, PKS 0304-392 and PKS 2044-168, show no evidence of its presence.

the line-corrected optical magnitude $\left(B_{\text {cor }}\right)$ given in Table 4. The observed quivalent widths of the emission lines and the resulting correction to the magnitude, derived as described in Wilkes et al. (1990), are also given in Table 4.

The model comparisons require an estimate of the ionizing luminosity of each quasar (1-1000 ryd). The monochromatic flux of the quasar at 1 ryd (rest frame) was estimated by extrapolating to 1 ryd $(912 \AA)$ from the observed optical magnitude, assuming a power law with an energy spectral index of
1.0. X-ray measurements are available for six of the 12 objects; the flux density at $1.0 \mathrm{keV}$ and effective slope from 1 ryd to 0.6 $\mathrm{keV}$ are given in Table 4. When X-ray detection was available, the mean value of the effective slope for the rest of the sample was utilized. The ionizing luminosity $\left(L_{\text {ion }}\right)$ was then determined using the following continuum shape:

\begin{tabular}{ccc}
\hline \hline $\begin{array}{c}\text { Energy Spectral } \\
\text { Index }\end{array}$ & $\begin{array}{c}\text { Low-Energy } \\
\text { Boundary }\end{array}$ & $\begin{array}{c}\text { High-Energy } \\
\text { Boundary }\end{array}$ \\
\hline Effective slope $\ldots \ldots \ldots \ldots$ & $1 \mathrm{ryd}$ & $0.6 \mathrm{keV}$ \\
$\alpha=1.0 \ldots \ldots \ldots \ldots \ldots \ldots$ & $0.6 \mathrm{keV}$ & $5.0 \mathrm{keV}$ \\
$\alpha=0.7 \ldots \ldots \ldots \ldots \ldots$ & $5.0 \mathrm{keV}$ & $13.6 \mathrm{keV}$ \\
\hline
\end{tabular}

The true shape of the UV/X-ray continuum is, as yet, unknown. The shape chosen here represents a reasonable compromise between the extremes of a blackbody turnover at energies just higher than the $I U E$ observed band and at energies just lower than the soft X-ray. A flattening toward harder $\mathrm{X}$-ray energies is included to allow for the presence of a reflected component as seen in Ginga spectra of AGNs (Pounds et al.

TABLE 4

OBSERVED AND INTRINSIC FLUXES AND LUMINOSITIES

\begin{tabular}{|c|c|c|c|c|c|c|c|c|c|c|}
\hline \multirow[b]{2}{*}{ ОВJECT } & \multicolumn{4}{|c|}{$W_{\lambda}^{\mathrm{a}}(\AA)$} & \multirow[b]{2}{*}{$\Delta B$} & \multirow[b]{2}{*}{$B_{\text {cor }}$} & \multirow{2}{*}{$\begin{array}{c}L_{912}{ }^{\mathrm{b}} \\
\left(\mathrm{ergs} \mathrm{s}^{-1}\right)\end{array}$} & \multirow[b]{2}{*}{$F_{1 \mathrm{kev}}{ }^{\mathrm{c}}$} & \multirow[b]{2}{*}{$\alpha_{u v x}^{d}$} & \multirow{2}{*}{$\begin{array}{c}L_{\text {ion }}{ }^{\mathrm{e}} \\
\left(\text { ergs s }^{-1}\right)\end{array}$} \\
\hline & O vi & Ly $\alpha$ & $\mathrm{C}$ IV & C III] & & & & & & \\
\hline Q0000-398 & 151 & 622 & 224 & $\ldots$ & 0.54 & 18.8 & 1.9 & $\ldots$ & & 0.54 \\
\hline Q0207-398 ...... & 38 & 446 & 75 & $\ldots$ & 0.35 & 17.70 & 5.2 & 3.2 & 2.0 & 1.8 \\
\hline PKS $0304-392 \ldots \ldots \ldots \ldots$ & $\ldots$ & 197 & 62 & 52 & 0.05 & 17.6 & 3.0 & $\ldots$ & $\ldots$ & 0.85 \\
\hline Q1101-264 ............. & $\ldots$ & 209 & 78 & 43 & 0.08 & 16.16 & 14 & 7.8 & 2.2 & 4.0 \\
\hline Ton $1530 \ldots \ldots \ldots \ldots \ldots$ & $\ldots$ & 236 & 30 & $\ldots$ & 0.05 & 15.54 & 22 & 6.9 & 2.4 & 5.3 \\
\hline PG $1247+265 \ldots \ldots \ldots \ldots \ldots$ & $\ldots$ & 243 & 82 & $\ldots$ & 0.07 & 15.60 & 20 & $\ldots$ & $\ldots$ & 5.6 \\
\hline PKS $1331+170 \ldots \ldots \ldots \ldots$ & $\ldots$ & 205 & 51 & $\ldots$ & 0.05 & 16.89 & 6.5 & 4.9 & 2.1 & 2.0 \\
\hline Mrk $679 \ldots \ldots \ldots \ldots \ldots \ldots$ & $\ldots$ & 230 & 44 & $\ldots$ & 0.04 & 17.01 & 5.0 & $\ldots$ & $\ldots$ & 1.5 \\
\hline $1559+089 \ldots \ldots \ldots \ldots \ldots \ldots$ & $\ldots$ & 240 & 51 & 50 & 0.21 & 16.9 & 7.4 & 7.2 & 2.0 & 2.2 \\
\hline PKS 2044-168 ......... & $\ldots$ & 448 & 101 & 62 & 0.09 & 17.64 & 2.9 & $\ldots$ & $\ldots$ & 0.82 \\
\hline Q2204-408 $\ldots \ldots \ldots \ldots \ldots$ & $\ldots$ & 354 & 87 & $\ldots$ & $0.27^{\mathrm{c}}$ & 17.84 & 5.5 & $<2.5$ & $>2.0$ & 1.6 \\
\hline PKS 2212-299 .......... & 35 & 275 & 65 & $\ldots$ & 0.25 & 17.83 & 4.2 & $\ldots$ & $\ldots$ & 1.1 \\
\hline Defaults $^{\mathrm{f}} \ldots \ldots \ldots \ldots \ldots \ldots$ & 15 & 84 & 32 & 17 & $\ldots$ & $\ldots$ & $\ldots$ & $\ldots$ & 2.2 & $\ldots$ \\
\hline
\end{tabular}

a Observed equivalent widths for O vi $\lambda 1034$, Ly $\alpha \lambda 1215 \mathrm{~N}$ v $\lambda 1240, \mathrm{C}$ IV $\lambda 1549, \mathrm{C}$ III] $\lambda 1909$.

${ }^{b}$ Intrinsic monochromatic luminosity at $912 \AA$ (rest frame), in units of ergs s ${ }^{-1} \mathrm{~Hz}^{-1}$.

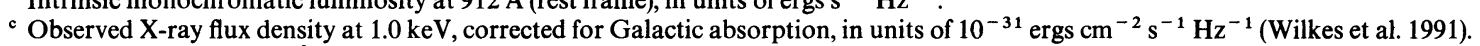

d Effective slope from $912 \AA$ to $0.6 \mathrm{keV}$ in the quasar rest frame.

e Total ionizing luminosity, in units of $10^{47} \mathrm{ergs} \mathrm{s}^{-1}$, integrated from 1 ryd $(912 \AA, 13.6 \mathrm{eV})$ to $1000 \mathrm{ryd}(13.6 \mathrm{keV})$ in the quasar rest frame assuming the spectrum described in the text $\left(H_{0}=50 \mathrm{~km} \mathrm{~s}^{-1} \mathrm{Mpc}^{-1}, q_{0}=0\right)$.

f When no measurement was available, these mean rest-frame values were adopted (Wilkes 1986) for equivalent widths and $\alpha_{\text {uvx }}$. 

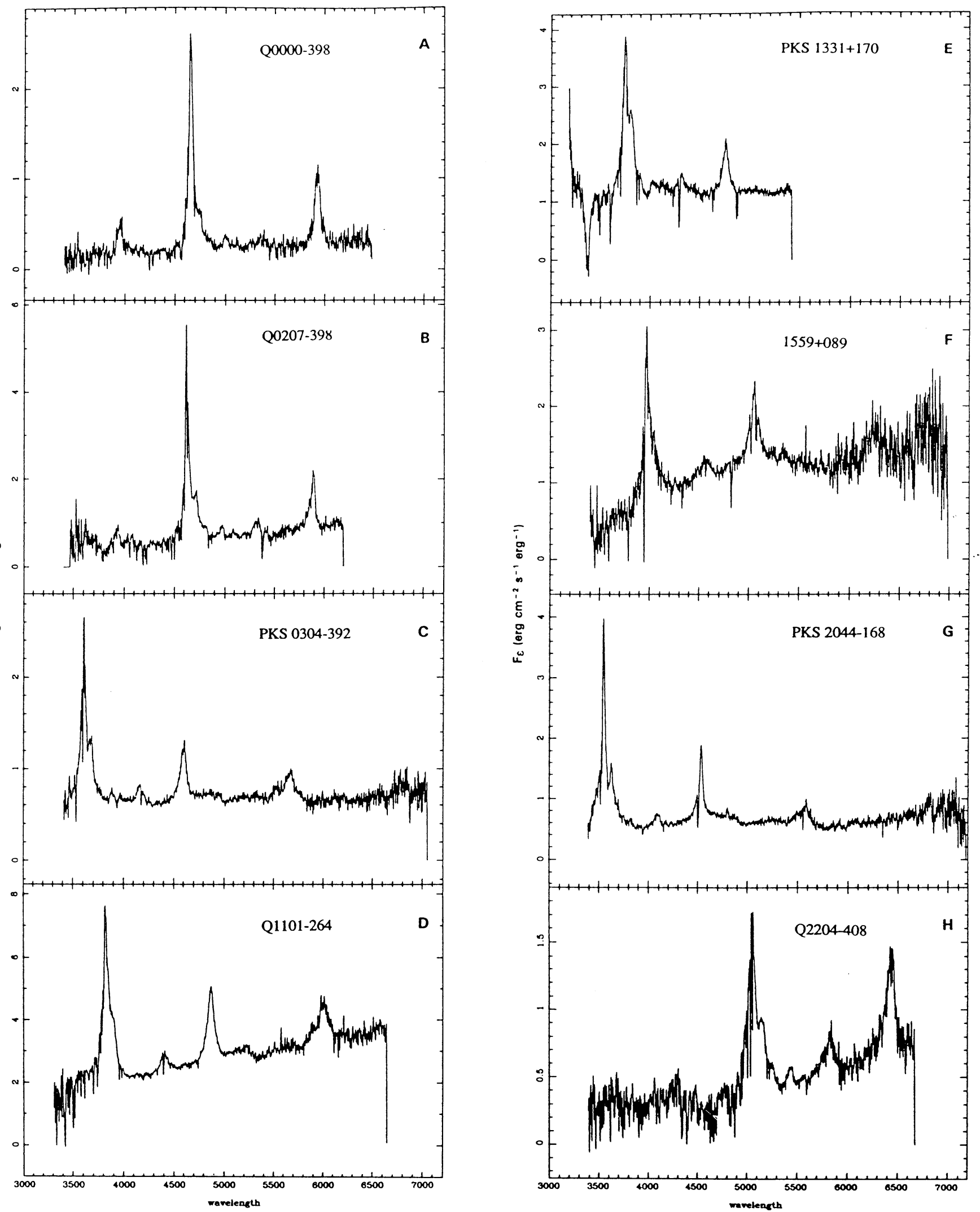

Fig. 1.-High-resolution spectra of all quasars, on a normalized $F_{\varepsilon}$ scale (ergs $\left.\mathrm{cm}^{-2} \mathrm{~s}^{-1} \mathrm{ergs}^{-1}\right)$. (a) Q0000-398; (b) Q0207-398; (c) PKS 0304-392; (d) Q1101-264; (e) PKS 1331+170; $(f) 1559+089 ;(g)$ PKS 2044-168; (h) Q2204-408; (i) PKS 2212-299; (j) Ton 1530, (k) Mrk 679; (l) PG 1247+265. 


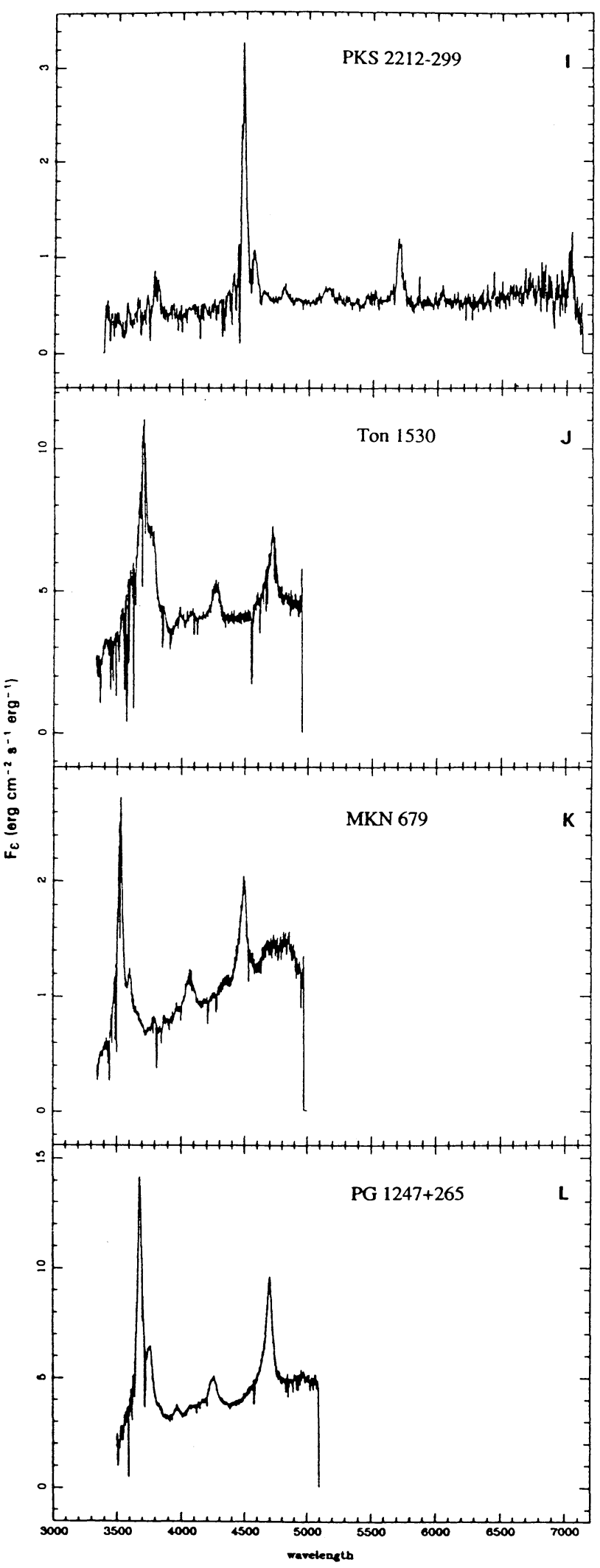

FIG. 1-Continued
1990); however, this region accounts for a small fraction (a few percent) of the ionizing flux, so the exact slope is not critical.

\section{THE MODEL}

In the following section we present the results of fitting model profiles to the data described in $\S 3$. The model profiles embody the principles and employ the assumptions described in $\S 2$. The chief ingredient missing from the presentation in $\S 2$ is the choice of dynamical model. As has already been mentioned, there are a variety of plausible dynamical models which can be used when constructing model profiles. These include outflows driven by radiation pressure with optically thin (e.g., Mathews 1974) or optically thick (Mathews 1986) clouds, outflows driven by relativistic particles (e.g., Weymann et al. 1982), infall with X-ray heating (Krolik \& London 1983), isothermal infall (Kwan \& Carroll 1982), gas-pressure-driven winds or radiation-pressure-driven winds (e.g., Shlosman, Vitello, \& Shaviv 1985). Ideally we would like to try as many of these as possible in an attempt to distinguish which are or are not capable of fitting the data. However, there exist enough free parameters within each of these that we have investigated only a single dynamical model in this paper. It is our intention to study other possible models in future work.

Our initial choice of dynamical model is that of freely falling gas. Under this assumption the gas velocity is given by

$$
v(r)=(2 G M / r)^{1 / 2}=9400 \mathrm{~km} \mathrm{~s}^{-1} M_{10}^{1 / 2} r_{\mathrm{pc}}^{-1 / 2},
$$

where $M_{10}$ is the central mass in units of $10^{10} M_{\odot}$ and $r_{\mathrm{pc}}$ is the radius in units of parsecs. We also assume that the ionizing luminosity is directly proportional to the accretion rate with some efficiency $\eta$.

One consequence of the connection between the accretion rate and the BELR pressure and ionization parameter is that the accretion efficiency must be low in order to produce conditions favorable for line emission in clouds of the desired velocity. That is, to obtain an ionization parameter $\Xi \sim 1$ and cloud velocities $v \sim 1000 \mathrm{~km} \mathrm{~s}{ }^{-1}$, the efficiency must be $\eta \sim$ $10^{-3} \Xi T_{8} / v_{8}$, where $v_{8}$ is velocity in units of $1000 \mathrm{~km} \mathrm{~s}^{-1}$ and $T_{8}$ is hot phase temperature in units of $10^{8} \mathrm{~K}$ (cf. eqs. [4] and [5] and Krolik \& London 1983). Another consequence of the infall assumption is that the illuminated cloud faces are moving toward us, so that the line asymmetries, which manifest themselves as blue excesses in the observed data, must result from absorption within the clouds rather than from intercloud absorption. As shown by Wilkes (1984) and Wilkes \& Carswell (1982), and as will be further demonstrated in the following section, the line which is most obviously asymmetric in the spectra we fit is C IV 21549 . Since this line is expected to be no more asymmetric than $\mathrm{Ly} \alpha$, and since clouds with large column densities are a likely mechanism for producing such asymmetries within the framework of our model assumptions, we will assume that the cloud radiation pattern is the same for all the various lines when constructing our models. Specifically, we assume that the model clouds radiate isotropically in the hemisphere from which they are illuminated, and according to a cosine dependence in the unilluminated hemisphere:

$$
R(\theta)=\min (1,1+\cos \theta),
$$

where $\theta$ is the angle between the observer's line of sight to the cloud and the radius vector between the cloud and the ionizing source. This radiation pattern is consistent with the assumption that each area element on the cloud's surface radius iso- 
tropically if it is illuminated. Although this is more likely to be correct for lines such as C III] $\lambda 1909$ and $N$ v $\lambda 1240$, we adopt it for all lines.

We also neglect the effects of electron scattering by the hot phase gas (Kallman \& Krolik 1986; Shields \& McKee 1981) in order to understand better the influence of the many other quantities on the profile shapes. This turns out to be justified a posteriori by the fact that the hot phase Thomson depth is generally small $(\leq 1)$ in our best-fit models.

A summary of the assumptions inherent in our models is as follows: spherical symmetry for the cloud spatial distribution and for the ionizing continuum radiation; conservation of hot gas mass flux within the BELR; the use of a cloud radiation pattern which is the same for all lines, and which corresponds to clouds being totally opaque to all line photons in their interiors; and radial cloud motions relative to the compact object with free-fall velocity distribution.

The free parameters which we can adjust in an attempt to fit the data are (1) accretion efficiency; (2) central mass; (3) and (4) minimum and maximum radii (i.e., distance from central object) at which clouds are assumed to exist; (5) hot phase temperature normalization $\left(T_{0}\right.$ in eqs [6] and [7]); (6) hot phase temperature distribution ( $\delta$ in eqs. [6] and [7]); (7) covering fraction normalization $\left(C_{0}\right.$ in eq. [9]); (8) covering fraction distribution ( $\epsilon$ in eq. [9]); (9) photoionization model parameters (ionizing spectrum, cloud column and element abundances).

Based on the discussion in $\S 2.7$, we can isolate which of these parameters most affect the synthetic profiles and which are truly independent. According to equations (6), (7), and (8), the accretion efficiency and fiducial hot phase temperature always appear in the ratio $\eta / T_{0}$. The mass of the central object only enters through the velocity equation, and so always appears as a ratio with the fiducial radius, $M / r_{0}$. However, $r_{0}$ remains independent as it affects the pressure scale. Therefore, from equations (10-(12) we conclude that the free parameters which will most affect our model profiles away from the extreme wings (where the continuum location introduces significant errors) are (1) the covering fraction normalization, $C_{0}$, which affects the total line strength; (2) the covering fraction distribution, $\epsilon$, which affects the line shape; and (3) the ratio $\eta / T_{0}$, which affects the ionization parameter and pressure scale and thereby the relative strengths of various lines. Other parameters which are important are $r_{0}$ and the ratio $M / r_{0}$, which determines the line width. The parameters $\delta$ and $r_{\max }$ affect the relative shapes of various lines and the "sharpness" of the peak at line center, respectively, neither of which is severely constrained in most of our sample. The dependence of the line fluxes emitted by the clouds (cf. eq. [8]) on the photoionization model parameters will be discussed separately in the next section.

The procedure we use for fitting the data to synthetic profiles is as follows: $(a)$ initial choice of the relevant free parameter values; (b) construction of a spatial grid of 50-100 logarithmically spaced points spanning the range of allowed radii; $(c)$ calculating the dynamical quantities (velocity, pressure, ionization parameter, etc.) along the grid; $(d)$ rebinning the data on a wavelength scale which corresponds to the velocity grid in the vicinity of each line; $(e)$ calculation of the synthetic profiles of the various lines by numerically integrating equation $(2) ;(f)$ use of an automated minimization algorithm (the "simplex method;" Press et al. 1986), and repetition of steps $c-e$ to find the best fit of the synthetic profile to the observed data of each object. All of the strong emission lines from each object are fitted simultaneously, using the same set of model parameters and the same fitting algorithm.

The minimization is achieved by varying the covering factor normalization $C_{0}$ (cf. eq. [9]), the accretion efficiency $\eta$, and the minimum accessible radius $r_{\min }$. The ratio of the minimum and maximum radii and the ratio of the central object mass to the minimum radius (i.e., the range of accessible velocities) are held constant during this procedure. These ratios, together with other parameters, such as the logarithmic derivatives of covering fraction and ICM temperature, are varied "by hand" in order to improve the fit. The quality of the fit of a given synthetic profile to the observed data is judged by the value of $\chi^{2}$ calculated between the observed and model distributions:

$$
\chi^{2}=\sum_{i=1}^{n_{\mathrm{bins}}} \omega_{i}\left(F_{\mathrm{obs}, i}-F_{\mathrm{model}, i}\right)^{2}
$$

where $F_{\mathrm{obs}, i}$ and $F_{\text {model, } i}$ are the observed and model profiles, respectively. The weight factor is given by $\omega_{i}=1$ if $F_{\mathrm{obs}, i} \geq \sigma_{i}$ and $\omega_{i}=0$ otherwise, and $\sigma_{i}$ is the error estimate on the observed data based on the uncertainty in the continuum placement. This weighting is chosen in order to place emphasis on wavelength bins where the line strength exceeds the continuum by a significant amount. According to the definition, $\chi^{2}$ is similar to the familiar quantity, but it lacks the statistical meaning associated with the assumption that the errors on the data are normally distributed. Therefore, although we are confident that a minimum of $\chi^{2}$ does define a "best" fit, we cannot assign any probability of random occurrence to a given value of this quantity, nor can we intercompare the quality of the fits to the various objects in a rigorous way.

\section{RESULTS}

\subsection{Line Strengths}

Before fitting the detailed profile shapes, we compare the total strengths of the observed lines with the predictions of standard photoionization models. For most of the spectra in our sample the line fluxes have been estimated by using the C IV $\lambda 1549$ profile as a template to deblend $N \vee \lambda 1240$ from $\operatorname{Ly} \alpha$ (Wilkes 1984). Figure 2 shows a plot of the $N$ v $\lambda 1240 /$ Ly $\alpha$ and C IV $\lambda 1549 / \mathrm{Ly} \alpha$ ratios. The lowercase letters denote the measured values from the various objects in our sample, while the curves denote the photoionization model predictions. The solid curves correspond to models calculated at a given value of ionization parameter, ranging from $\log \Xi=-1$ to $\log \Xi$ $=0$, and the dashed curves are drawn along contours of constant pressure ranging from $\log P=-3.5$ to $\log P=-0.5$ (where $P$ is measured in dynes $\mathrm{cm}^{-2}$ ).

Table 5 summarizes the parameters of the photoionization models shown in the various panels of Figure 2: their ionizing spectra, column density and elemental abundances. The elemental abundances are chosen to be perturbations around those adopted by Kwan \& Krolik (1981), which are the same as the solar values of Trimble (1975) with carbon reduced by $40 \%$. The clouds are assumed to be semi-infinite slabs (approximating the column of $10^{25} \mathrm{~cm}^{-2}$ or greater, which we expect will be capable of producing the line asymmetries) with one exception. The ionizing spectra span the range from radioloud quasars with $\alpha_{\mathrm{ox}}=1.2$ (the "double power law") to Seyfert galaxies with a UV excess ("blue bump") to objects with a strong thermal component dominating the ultraviolet, 


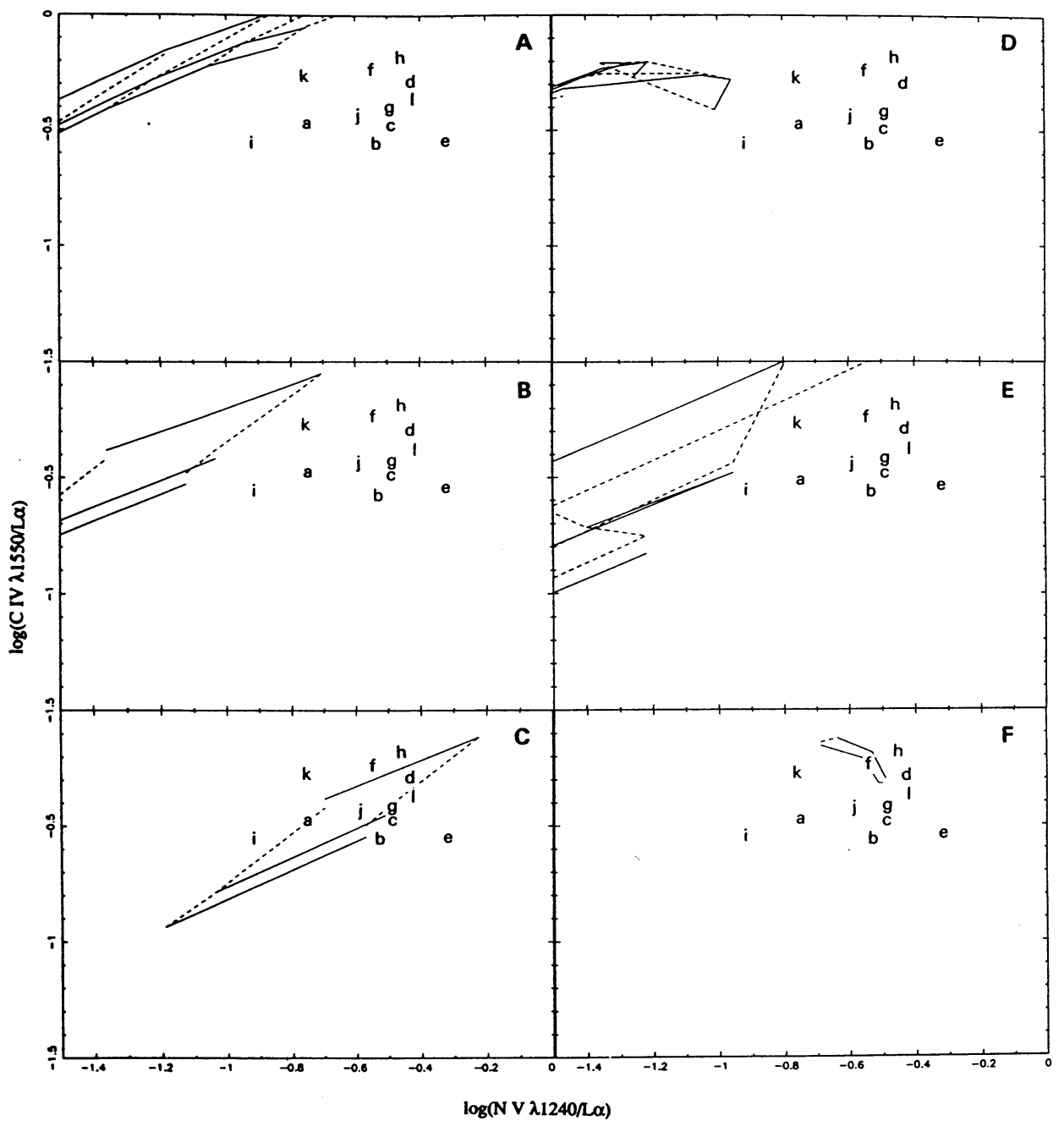

Fig. 2.-Location of photoionization models and observed objects in the two-line-ratio diagram $\log (\mathrm{C}$ IV $\lambda 1549 / \mathrm{Ly} \alpha)$ vs. $\log (\mathrm{N} v \lambda 1240 / \mathrm{Ly} \alpha)$. Letter symbols correspond to measured line ratios from the observed data: (a) Q0000-398; (b) Q0207-398; (c) PKS 0304-392; (d) Q1101-264; (e) PKS 1331+170; (f) 1559+089; (g) PKS 2044-168; (h) Q2204-408; (i) PKS 2212-299; (j) Ton 1530; (k) Mrk 679; (l) PG 1247+265. The panels correspond to models with various ionizing spectra, cloud column density, and abundance assumptions: Panels $a, b$, and $c$ have a hard double-power-law ionizing spectrum with $\alpha_{o x}=1.2$ (see Krolik \& Kallman 1988 for a description of this spectrum); panel $a$ models have the element abundances of Kwan \& Krolik (1981); panel $b$ has all metals suppressed by a factor of 3 ; and panel $c$ has nitrogen enhanced by a factor of 3 and carbon reduced by a factor of 2. Panel $d$ has an ionizing spectrum with a UV excess similar to that observed from NGC 5548 (see Krolik et al. 1990 for a description of this spectrum); panel $e$ has an ionizing spectrum with a large extreme ultraviolet excess (see Krolik \& Kallman 1988 for a description of the spectrum); and panel $f$ has the same spectrum as panel $d$, except with cloud column density reduced from $10^{23}$ to $10^{20}$ $\mathrm{cm}^{-2}$.

extreme ultraviolet, and soft X-ray bands (the "big bump"). These are defined in more detail in Krolik \& Kallman (1988).

For a given combination of these parameters the various line-ratio values correspond to various choices of ionization parameter and pressure. Since both ratios depend most strongly on ionization parameter, each family of models lies in a fairly narrow diagonal band in the diagram. The C IV $\lambda 1549 / \mathrm{Ly} \alpha$ ratio tends to decrease with increasing cloud pressure, owing to thermalization of the C IV $\lambda 1549$ line, while the $N$ v $\lambda 1240$ / $\mathrm{Ly} \alpha$ ratio is only very weakly affected by this process.

It is apparent from Figure 2 that the observed line-ratio values appear to lie near the boundary of the region accessible to the photoionization models we have considered, and in some cases are outside this region. Furthermore, the photoionization models which adhere most closely to the standard assumptions about elemental abundances and cloud column (panels $a, d$, and $e$ ) are clearly inconsistent with the observed line strengths.

The models which are more successful in accounting for the observed ratios are those in which nitrogen is enhanced, carbon is suppressed, or the cloud column is greatly reduced. All these models allow greater $N \mathrm{v} \lambda 1240 / \mathrm{Ly} \alpha$ ratios for given C IV $\lambda 1549 /$ Ly $\alpha$ ratios by enhancing the nitrogen-to-carbon ratio in the clouds. The most successful models, shown in panel $c$, are those in which the nitrogen abundance is enhanced by a factor of 3, while carbon and all other abundances are left at the Kwan \& Krolik (1981) values. In the case of the reduced column density models this occurs because C IV is more completely ionized than is the more durable $\mathrm{N}$ v. This is consistent with the results of past photoionization models, such as Baldwin \& Netzer (1978) and Kwan \& Krolik (1981), who showed that either N/C values greatly in excess of solar or an 
TABLE 5

Photolonization Model Parameters

\begin{tabular}{llll}
\hline \hline Panel & \multicolumn{1}{c}{ Spectrum } & \multicolumn{1}{c}{ Abundances } & $\begin{array}{c}\text { Cloud Column } \\
\text { Density }\end{array}$ \\
\hline$a \ldots \ldots$ & Double power law $^{\mathrm{a}}$ & $\mathrm{C}:$ solar $/ 2.5$ & Semi-infinite \\
$b \ldots \ldots$ & Double power law $^{\mathrm{a}}$ & Metals: solar $/ 3$ & Semi-infinite \\
$c \ldots \ldots$ & Double power law $^{\mathrm{a}}$ & $\mathrm{C}:$ solar $/ 2.5 ; \mathrm{N}:$ solar $\times 3$ & Semi-infinite \\
$d \ldots \ldots$ & Blue bump $^{\mathrm{b}}$ & $\mathrm{C}:$ solar $/ 2.5$ & Semi-infinite \\
$e \ldots \ldots$ & Big bump $^{\mathrm{c}}$ & $\mathrm{C}:$ solar $/ 2.5$ & Semi-infinite \\
$f \ldots \ldots$ & Blue bump $^{\mathrm{b}}$ & $\mathrm{C}:$ solar $/ 2.5$ & $10^{20} \mathrm{~cm}^{-2}$ \\
\hline
\end{tabular}

${ }^{\text {a }}$ Double-power-law spectrum (cf. Krolik \& Kallman 1988):

$$
F_{v}^{\text {DPL }} \propto \begin{cases}v^{-1.2}, & h v<2 \mathrm{keV}, \\ v^{-0.7}, & h v<2 \mathrm{keV} .\end{cases}
$$

${ }^{\text {b }}$ Blue bump spectrum (cf. Krolik \& Kallman 1988):

$$
F_{v}=F_{v}^{(\text {DPL) }}+F_{v}^{\text {(disk) }} \text {, }
$$

where

$$
F_{v}^{\text {(disk) }} \propto v^{1 / 3} \exp \left(-h v / k T_{b}\right)
$$

and $k T_{b}=10 \mathrm{eV}$ and the fluxes of the two components integrated from $13.6 \mathrm{eV}$ to $13.6 \mathrm{keV}$ are the same.

${ }^{c}$ Big bump spectrum (cf. Krolik \& Kallman 1988):

$$
F_{v}=F_{v}^{(\mathrm{DPL})}+F_{v}^{(\text {disk) }}
$$

and $k T_{b}=80 \mathrm{eV}$, and the two components are weighted so that there is 25 times as much flux, integrated from $13.6 \mathrm{eV}$ to $13.6 \mathrm{keV}$, in the disk spectrum as in the power law.

additional component of line-emitting gas with large ionization parameter and low column density is necessary to account for the observed nitrogen and carbon line strengths. The reduced column density models have the property that the enhancement in $N \vee \lambda 1240 /$ Ly $\alpha$ relative to $C$ IV $\lambda 1549 / \mathrm{Ly} \alpha$ depends on ionization parameter, since at low values there can exist a $\mathrm{C}$ IV ionization front within the cloud. The assumption of low cloud column is also not consistent with any cloud radiation asymmetry, so we will focus on the modified abundances in the rest of this paper.

Following a suggestion by Netzer (1977), we have also considered the effects of suppressing all the metals by a factor of 3 . This has the consequence of shifting some of the cloud cooling from the more abundant trace ions (e.g., C IV) to the less abundant (e.g., $\mathrm{N} \mathrm{v}$ ), but for the values adopted here the effect is too small to account for the observed line ratios.

We consider it worthy of some note that relatively unusual assumptions concerning abundances succeed where other plausible ones fail at explaining the data. However, one weakness of this analysis is in the use of Gaussian profiles for the lines when inferring their total fluxes. It is one of the goals of the detailed profile modeling to test whether differences in profile shapes which arise within the framework of the standard BELR model can help to bring a larger class of photoionization models into accord with the data. As pointed out in $\S 2$, the shape of the ionizing spectrum determines the temperature of the hot phase gas, $T_{\text {hot }}$. However, for spectra such as the double power law, the $T_{\text {hot }}$ value depends on the extrapolation to energies outside the range of direct observations, i.e., to $\geq 100 \mathrm{keV}$ and $\leq 0.1 \mathrm{eV}$. For plausible assumptions about this extrapolation, $T_{\text {hot }}$ can range from $10^{7}$ to $10^{8} \mathrm{~K}$; in what follows we will treat it as a free parameter. In what follows we fit the observed profiles to synthetic profiles calculated only with the grid of photoionization models shown in panel $c$, i.e., with the abundance of nitrogen enhanced and the abundance of carbon suppressed relative to solar values and using the hard double-power-law ionizing spectrum.
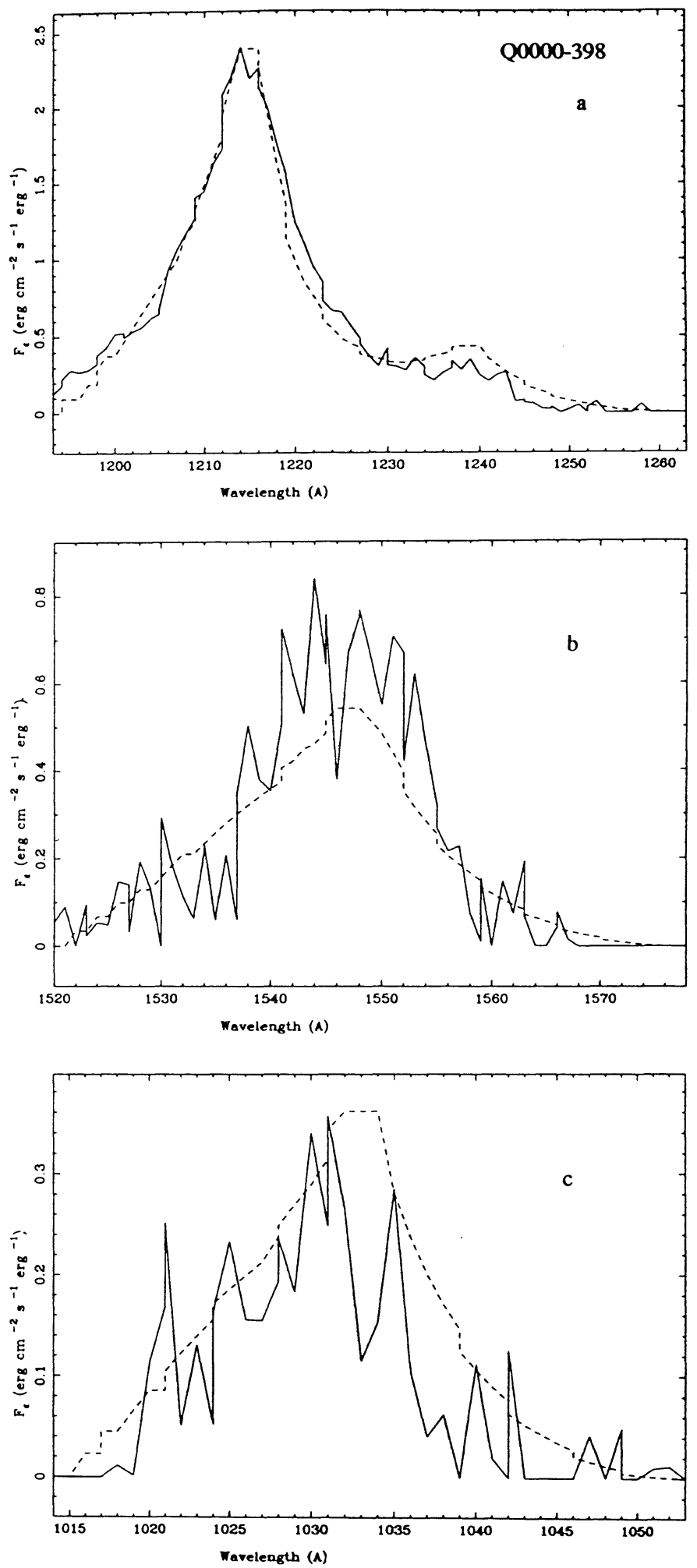

FIG. 3.-Comparison of observed (solid curve) and model (dashed curve) line profiles for the object Q0000-398. (a) Ly $\alpha / \mathrm{N} v \lambda 1240$ blend. (b) C IV $\lambda 1550$. (c) O vi $\lambda 1034$ line. 
TABLE 6

FitTing Parameters

\begin{tabular}{|c|c|c|c|c|c|c|c|c|}
\hline Object & $M^{\mathrm{a}}$ & $r_{\min }{ }^{b}$ & $r_{\max }{ }^{b}$ & $\eta^{\mathrm{c}}$ & $L_{e}^{\mathrm{d}}$ & $C_{0}{ }^{\mathrm{e}}$ & $\epsilon^{\mathrm{f}}$ & $\delta^{f}$ \\
\hline Q0000-398 & 1400 & 38 & 3800 & $2.40 \mathrm{E}-05$ & 5.4 & $7.50 \mathrm{E}-03$ & -7 & 0.5 \\
\hline Q0207-398 & $1 E+05$ & 3700 & 37000 & $5.20 \mathrm{E}-05$ & 18 & $1.70 \mathrm{E}-03$ & -6.8 & 0.5 \\
\hline PKS $0304-392 \ldots \ldots \ldots \ldots$ & 7700 & 200 & 20000 & $4.20 \mathrm{E}-05$ & 8.5 & $1.40 \mathrm{E}-03$ & -7.2 & 0.5 \\
\hline Q1101-264 $\ldots \ldots \ldots \ldots \ldots$ & 3300 & 88 & 880 & $3.90 \mathrm{E}-05$ & 40 & $1.40 \mathrm{E}-03$ & -7.2 & 0.5 \\
\hline Ton $1530 \ldots \ldots$ & 84000 & 2200 & $2 \mathrm{E}+05$ & $8.70 \mathrm{E}-05$ & 53 & $4.90 \mathrm{E}-04$ & -8 & 0.5 \\
\hline PG $1247+265$. & 20000 & 55 & 5500 & $8.20 \mathrm{E}-07$ & 56 & $1.20 \mathrm{E}-03$ & -6 & 1.5 \\
\hline PKS $1331+170 \ldots$ & 39000 & 1000 & 10000 & $1.00 \mathrm{E}-05$ & 2 & $6.00 \mathrm{E}-03$ & -6 & 2.4 \\
\hline Mrk $679 \ldots . .$. & 9200 & 240 & 24000 & $4.20 \mathrm{E}-05$ & 15 & $5.20 \mathrm{E}-04$ & -8 & 0.5 \\
\hline $1559+089 \ldots$ & 2400 & 63 & 6300 & $3.70 \mathrm{E}-05$ & 24 & $8.40 \mathrm{E}-03$ & -7 & 0.5 \\
\hline PKS 2044-168 .......... & 78000 & 2100 & 21000 & $6.10 \mathrm{E}-05$ & 8.2 & $1.20 \mathrm{E}-03$ & -6.8 & 0.5 \\
\hline Q2204-408 $\ldots \ldots \ldots \ldots \ldots$ & 2900 & 77 & 7700 & $3.60 \mathrm{E}-05$ & 16 & $2.60 \mathrm{E}-03$ & -8 & 0.5 \\
\hline PKS 2212-299 .......... & 33000 & 880 & 88000 & $2.40 \mathrm{E}-06$ & 11 & $1.10 \mathrm{E}-04$ & -6 & 1.5 \\
\hline
\end{tabular}

${ }^{\text {a }}$ Compact object mass $M$ has units $10^{8} M_{\odot}$.

b Minimum and maximum accessible radii are in units of pc.

c $\eta$ is the accretion efficiency.

${ }^{d} L_{c}$ is the continuum luminosity in units of $10^{46} \mathrm{ergs} \mathrm{s}^{-1}$.

e $C_{0}$ is the covering fraction at the minimum radius in units of $\mathrm{pc}^{-1}$.

f $\epsilon$ is the logarithmic derivative of the covering fraction with respect to velocity, and $\delta$ is the logarithmic derivative of hot phase temperature with respect to radius.

TABLE 7

Fit ERRORS

\begin{tabular}{|c|c|c|c|}
\hline Object & Error $^{\mathrm{a}}$ & $\chi^{2 \mathrm{~b}}$ & $n_{\text {bins }}$ \\
\hline Q0000-398 & 0.15 & 1.7 & 214 \\
\hline Q0207-398 & 0.12 & 9.8 & 216 \\
\hline PKS $0304-392 .$. & 0.1 & 1.1 & 200 \\
\hline Q1101-264 $\ldots \ldots \ldots \ldots \ldots \ldots$ & 0.22 & 19 & 213 \\
\hline Ton $1530 \ldots \ldots \ldots \ldots \ldots \ldots$ & 0.34 & 35 & 264 \\
\hline PG $1247+265 \ldots$ & 0.41 & 380 & 687 \\
\hline PKS $1331+170 \ldots \ldots \ldots \ldots$ & 0.16 & 8.5 & 156 \\
\hline Mrk $679 \ldots \ldots \ldots \ldots \ldots \ldots$ & 0.06 & 3.4 & 251 \\
\hline $1559+089 \ldots \ldots \ldots \ldots \ldots \ldots$ & 0.17 & 10 & 222 \\
\hline PKS 2044-168 .......... & 0.20 & 2.4 & 199 \\
\hline Q2204-408 $\ldots \ldots \ldots \ldots \ldots$ & 0.12 & 1.8 & 236 \\
\hline PKS $2212-299 \ldots \ldots \ldots \ldots$ & 0.01 & 0.17 & 285 \\
\hline
\end{tabular}

${ }^{\text {a }}$ Error estimate derived from continuum placement uncertainty, in units of $\mathrm{s}^{-1}$.

${ }^{b} \chi^{2}$ characterizing difference between data and best-fit model, according to eq. (12).

${ }^{c}$ Number of wavelength bins used in fit.

\subsection{Line Profiles}

The results of fitting to the line profiles can best be described by first discussing the fits to a spectrum which we consider to be unexceptional in its fitted parameters, and then comparing with it other spectra which illustrate the effects of variations in the parameters.

Figure 3 shows the spectrum of Q0000-398 in the vicinity of the strongest emission lines. The data after continuum subtraction are shown as the solid curve, and the model is shown as the dashed curve. As shown in the figure, the agreement between the data and the model is within the errors associated with both the data and the continuum fitting. Table 6 gives the values of the various fitting parameters derived for Q0000 - 398 and the other objects in our sample, and Table 7 gives the quantities associated with error estimation. These include the error estimate derived from uncertainty in the continuum placement (cf. $\S 3$ ); the $\chi^{2}$ of the fit, defined in equation (14); and the number of wavelength bins used in the fit.

Table 8 gives the values of some of the other associated

TABLE 8

DeRIVED Broad-Line Region QuANTITIES

\begin{tabular}{|c|c|c|c|c|c|c|c|c|}
\hline Object & $\begin{array}{l}\log v^{\mathrm{a}} \\
(\max )\end{array}$ & $\begin{array}{l}\log v^{a} \\
(\min )\end{array}$ & $\begin{array}{c}\log \Xi^{b} \\
(\max )\end{array}$ & $\begin{array}{c}\log P^{c} \\
(\max )\end{array}$ & $\begin{array}{c}\log \Xi^{b} \\
(\min )\end{array}$ & $\begin{array}{c}\log P^{c} \\
(\mathrm{~min})\end{array}$ & $C_{\text {tot }}^{\mathrm{d}}$ & $\tau_{\mathrm{Th}}{ }^{\mathrm{e}}$ \\
\hline Q0000-398 & 0.61 & -0.4 & -0.93 & -4 & -0.93 & -7.02 & 0.09 & 0.4 \\
\hline Q0207-398 ...... & 0.61 & -0.7 & -0.59 & -7.8 & -0.59 & -8.53 & 0.03 & 0.005 \\
\hline PKS 0304-392 & 0.62 & -1.2 & -0.68 & -5.5 & -0.68 & -7.73 & 0.02 & 0.06 \\
\hline Q1101-264 ......... & 0.61 & -1.2 & -0.72 & -4.1 & -0.72 & -6.31 & 0.02 & 0.8 \\
\hline Ton $1530 \ldots \ldots \ldots \ldots \ldots \ldots$ & 0.61 & -1.2 & -0.37 & -7.1 & -0.37 & -9.33 & 0.006 & 0.02 \\
\hline PG $1247+265 \ldots \ldots \ldots \ldots$ & 1.1 & -1.2 & -1.9 & -2.4 & -3.9 & -4.08 & 0.03 & 26 \\
\hline PKS $1331+170 \ldots \ldots \ldots \ldots$ & 0.62 & -0.7 & -1.3 & -6.9 & -3.2 & -7.63 & 0.1 & 0.01 \\
\hline Mrk $679 \ldots \ldots \ldots \ldots \ldots$ & 0.61 & -1.2 & -0.68 & -5.4 & -0.68 & -7.64 & 0.007 & 0.1 \\
\hline $1559+089 \ldots \ldots \ldots \ldots \ldots$ & 0.61 & -1.2 & -0.74 & -4 & -0.74 & -6.22 & 0.01 & 0.7 \\
\hline PKS 2044-168 ......... & 0.61 & -0.7 & -0.53 & -7.7 & -0.53 & -8.45 & 0.02 & 0.003 \\
\hline Q2204-408 $\ldots \ldots \ldots \ldots$ & 0.61 & -1.2 & -0.75 & -4.3 & -0.75 & -6.56 & 0.03 & 0.4 \\
\hline PKS 2212-299 .... & 0.61 & -1.2 & -1.93 & -5.4 & -3.93 & -7.66 & 0.003 & 0.3 \\
\hline
\end{tabular}

a Logarithm of velocities at minimum and maximum radii, in units of $1000 \mathrm{~km} \mathrm{~s}^{-1}$.

b Logarithm of ionization parameter at minimum and maximum radii.

c Logarithm of gas pressure at minimum and maximum radii in units of dynes $\mathrm{cm}^{-2}$

d Total integrated covering fraction.

e Thomson optical depth through hot phase gas. 
quantities which depend on the fitting parameters, such as the range of ionization parameter, pressure, and velocity, along with the derived cloud covering fraction and the hot phase Thomson optical depth. This shows that the Thomson depths are all less than unity, with one exception which is discussed below. At the larger values given in the table, $0.1 \leq \tau_{\mathrm{Th}} \leq 1$, there will be a small quantitative effect of electron scattering on the Ly $\alpha$ line profile asymmetry. However, as demonstrated by Kallman \& Krolik (1986), this effect is not sufficient to suppress the line asymmetry for a free-fall model. This stands in contrast to the case of an accelerated outflow, $v \sim r$, for which electron scattering suppresses the asymmetry almost completely at these parameter values.

Figure 3 demonstrates that the line ratios are reproduced by the photoionization models, consistent with the location of Q0000-398 in the two-line-ratio diagram discussed in the previous subsection. The model lines are asymmetric; the apparent symmetry of the observed Ly $\alpha$ line is provided in part by the filling in of the red wing by blending with $N \vee \lambda 1240$. The greatest disparity between model and data occurs in the red wing of $\operatorname{Ly} \alpha$, where the model falls below the data, suggesting that lines with a different radiation pattern may give a better fit than that shown here.

As a measure of the sensitivity of our model fits to the data, we show in Figure 4 contours of constant $\log \chi^{2}$ for various values of two of the most important fitting parameters, the minimum accessible radius and the accretion efficiency. The third parameter used in the automated fitting, the covering fraction normalization, affects only the overall normalization of the model profile. It is held fixed at the best-fit value when deriving these contours. It is clear from this figure that the minima in the values of $r_{\min }$ and $\eta$ are well defined over the range of values considered here.

The results of fitting the other 11 objects, shown in Figures 5-15, are similar to those for Q0000-398. Most of the fits are of comparable quality, although there are a few notable exceptions. Many of the weaker lines in the data, such as C III] $\lambda 1909$ and $\mathrm{O}$ vI $\lambda 1034$, are fitted by the models, although the noise in the data prevents as precise a test of the fits as is possible for Ly $\alpha$ and $C$ IV $\lambda 1549$. Examination of Tables 6 and 8 show that

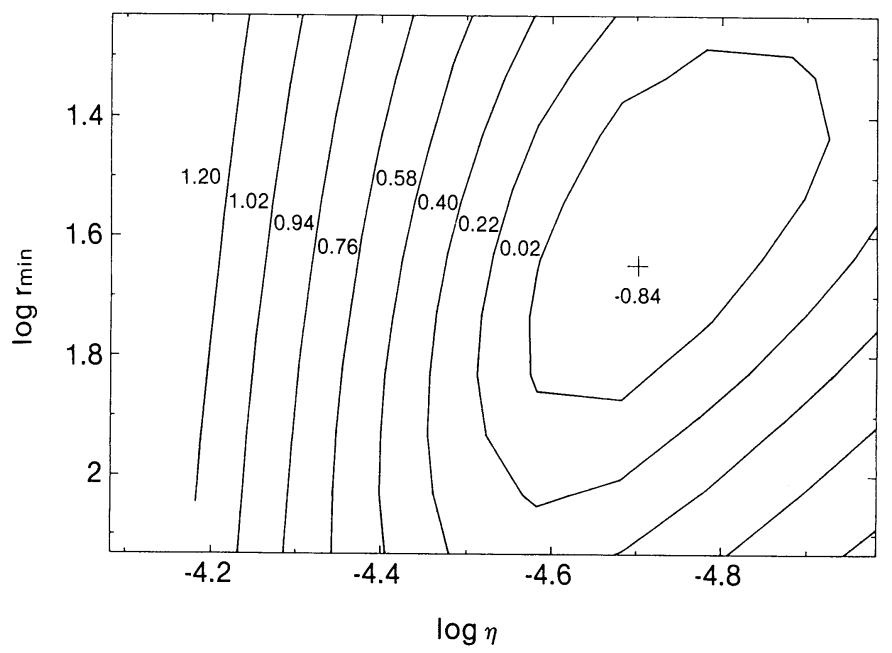

FIG. 4.-Contours of constant $\log \chi^{2}$ for various values of the minimum accessible radius and the accretion efficiency for the object Q0000-398. Contours are labeled with $\log \chi^{2}$. the fitted parameters cluster fairly close together in value, with one or two notable exceptions for each parameter. We now discuss these in turn.
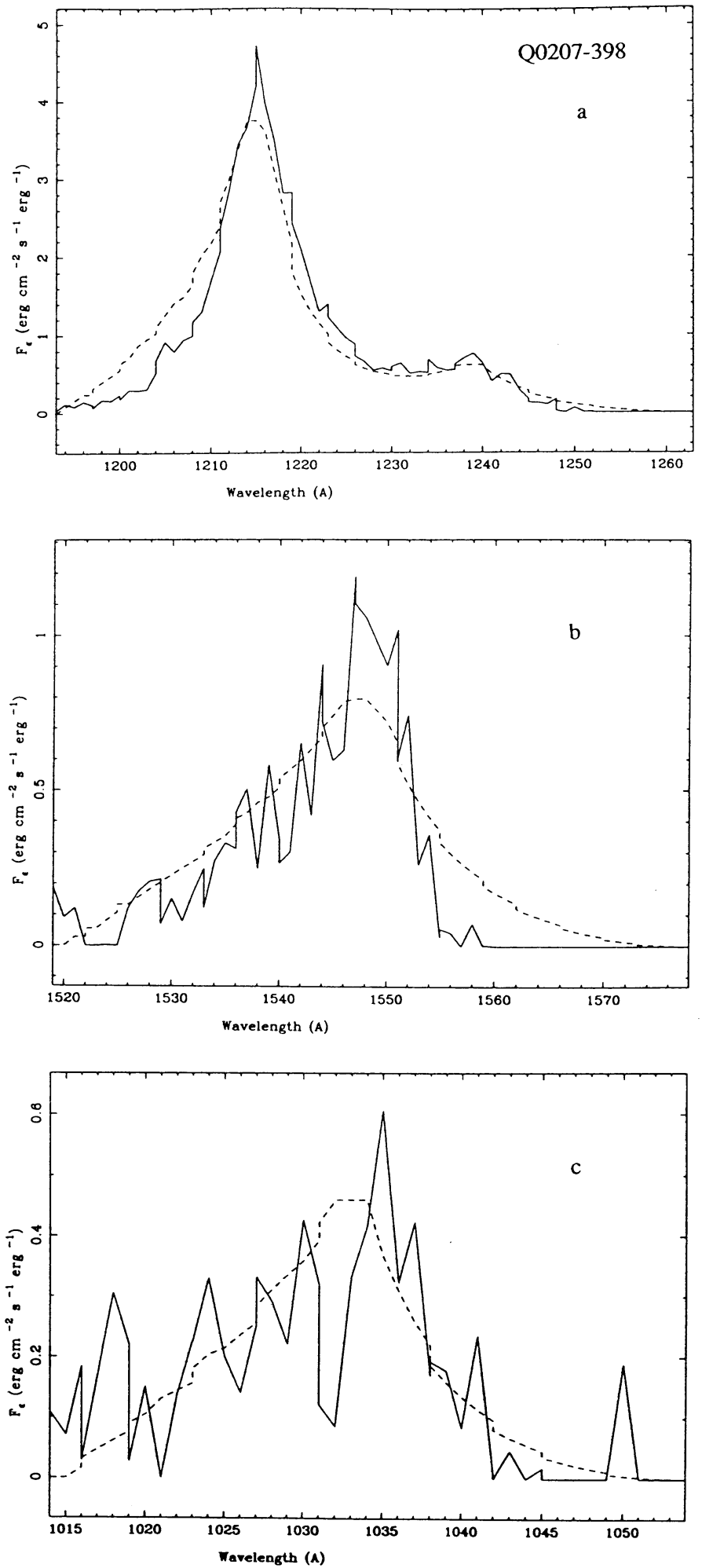

FIG. 5.-Comparison of observed (solid curve) and model (dashed curve) line profiles for the object Q0207-398. (a) Ly $\alpha / \mathrm{N} v \lambda 1240$ blend. (b) $\mathrm{C}$ IV $\lambda 1550$. (c) $O$ vi $\lambda 1034$ line. 

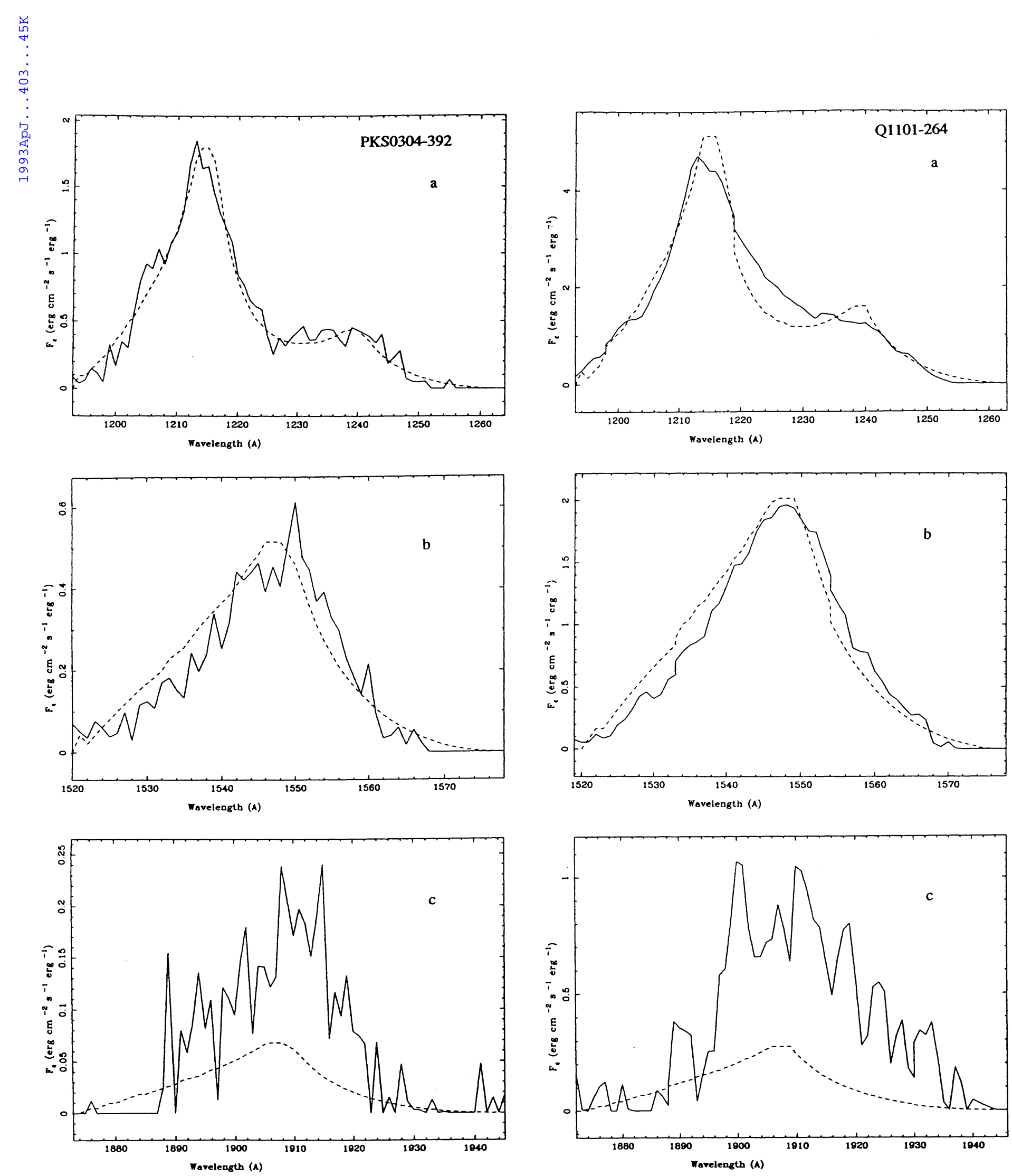

Fig. 6.-Comparison of observed (solid curve) and model (dashed curve) line profiles for the object PKS $0304-392$. (a) Ly $\alpha / \mathrm{N}$ v $\lambda 1240$ blend. (b) C IV $\lambda 1550$. (c) C III] $\lambda 1909$ line.

FIG. 7.-Comparison of observed (solid curve) and model (dashed curve) line profiles for the object Q1101-264. (a) Ly $\alpha / \mathrm{N} v \lambda 1240$ blend. (b) C IV $\lambda 1550$. (c) C III] 21909 line. 

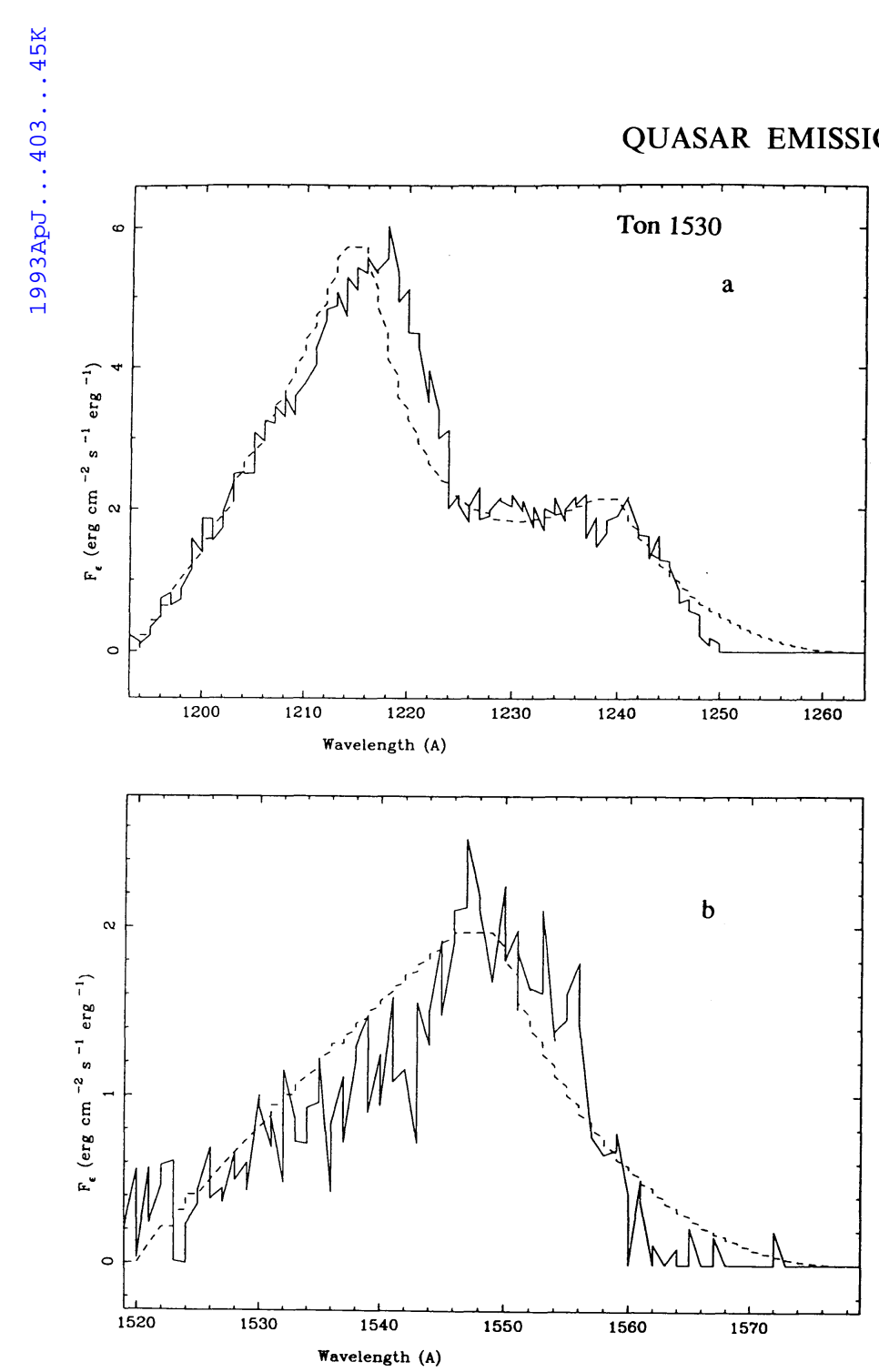

Fig. 8. - Comparison of observed (solid curve) and model (dashed curve) line profiles for the object Ton 1530. (a) $\mathrm{Ly} \alpha / \mathrm{N} \vee \lambda 1240$ blend. (b) C IV $\lambda 1550$.

\subsubsection{Velocity Law: Line Width}

One measure of the maximum cloud velocity is the width of the line near where the line and continuum merge, i.e., the "full width at zero intensity." In our fits this quantity is manifest as the value of $v_{\max }$ given in Table 8 . The greatest value of $v_{\max }$ occurs for the objects PG $1247+265$ and Mrk 679, reflecting the fact that the $\operatorname{Ly} \alpha$ line is significantly broader for these objects than for the others. Conversely, the minimum accessible velocity affects the width of the lines near their maximum flux, and is the same for all of our objects except for PKS $1331+170$ and PG $1247+265$. For these objects the lines are significantly less "peaky" than the others; it may be notable that PKS $1331+170$ also has the lowest ionizing luminosity and the greatest cloud covering factor of our sample.

For several objects our fits imply very large outer radii for the line-emitting region. At these large radii, the pressures are very low, and, if the cloud column densities are as large as we assume in the higher pressure regions of the BELR, the cloud sizes must be $D \sim 100$ pc $N_{25} T_{4} P_{-7}$, where $N_{25}$ is the cloud column density in units of $10^{25} \mathrm{~cm}^{-2}, T_{4}$ is the mean cloud temperature in units of $10^{4} \mathrm{~K}$, and $P_{-7}$ is the intercloud pressure in units of $10^{-7}$ dynes $\mathrm{cm}^{-2}$. Thus the cloud sizes are a significant fraction of the radius of the emission region. The

size of the emission may exceed the most probable size for the host galaxy itself.

However, we do not consider these results to be sufficiently secure to warrant debate of the customary assumptions about quasar environments and flows outside the BELR, since the fits are not extremely sensitive to the minimum accessible velocity (we estimate that our best-fit values of $r_{\max }$ an uncertain by a factor of 2-5) and since the more important physical quantity is the minimum accessible velocity, which may not be dominated by free-fall dynamics below $1000 \mathrm{~km} \mathrm{~s}^{-1}$. Most important, the differential covering fraction, $d C / d r$, is a very steeply decreasing function of $r$, and so only a small fraction of the total line emission comes from such large radii.

\subsubsection{Hot Phase Temperature}

All of our fits yield the same hot phase temperature at the fiducial (innermost) radius, $T_{0}=10^{7} \mathrm{~K}$. The majority also are consistent with a distribution of hot phase temperature which increases in proportion to $r^{1 / 2}$, so that the ionization parameter is constant throughout the BELR. We emphasize that this choice is the one that we deem simplest, and the one that was attempted first in our fitting procedure. Thus, these data may be consistent with other choices of hot phase temperature dis-
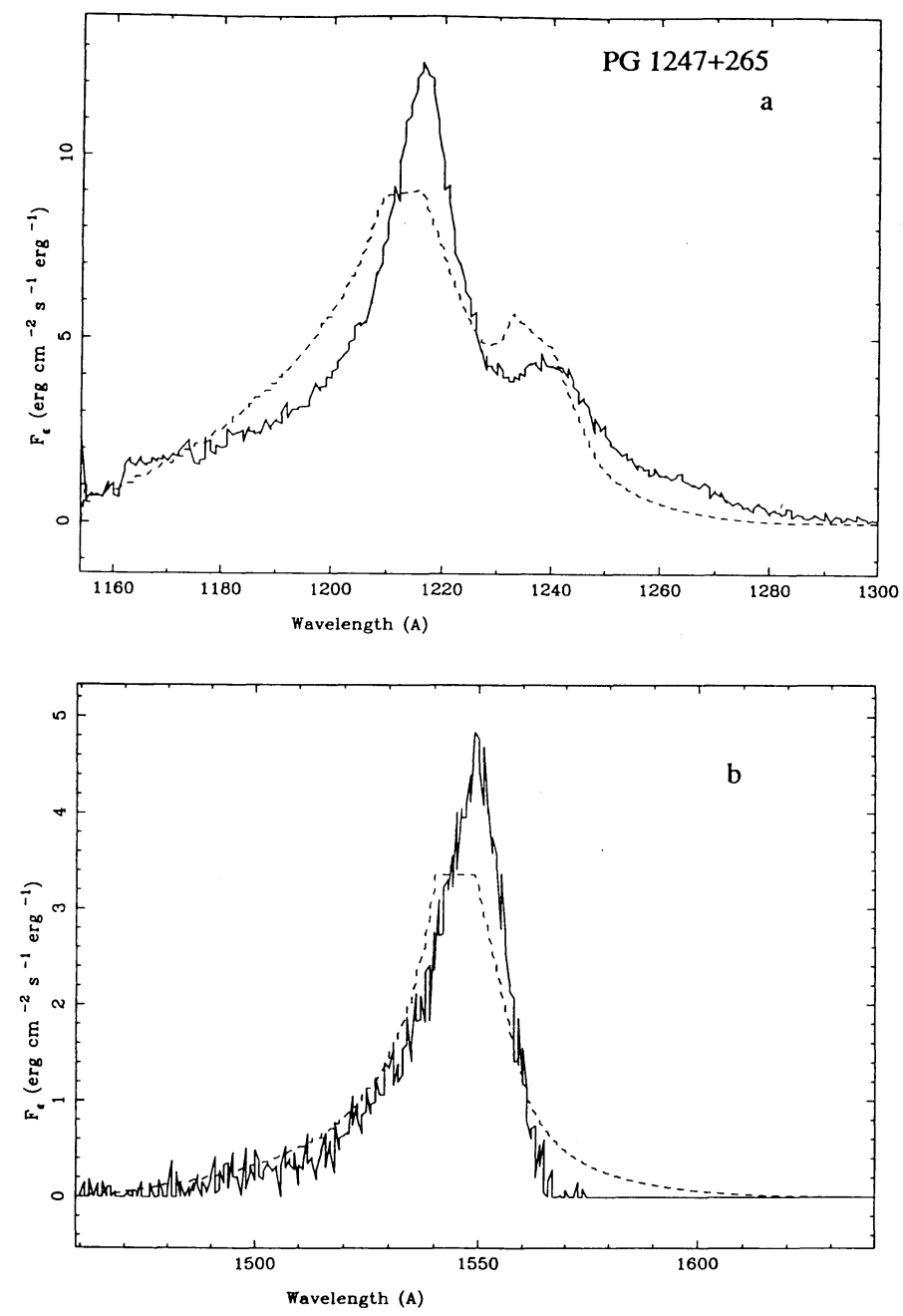

Fig. 9.- Comparison of observed (solid curve) and model (dashed curve) line profiles for the object PG $1247+265$. (a) Ly $\alpha / \mathrm{N} v \lambda 1240$ blend. (b) $\mathrm{C}$ IV $\lambda 1550$. 

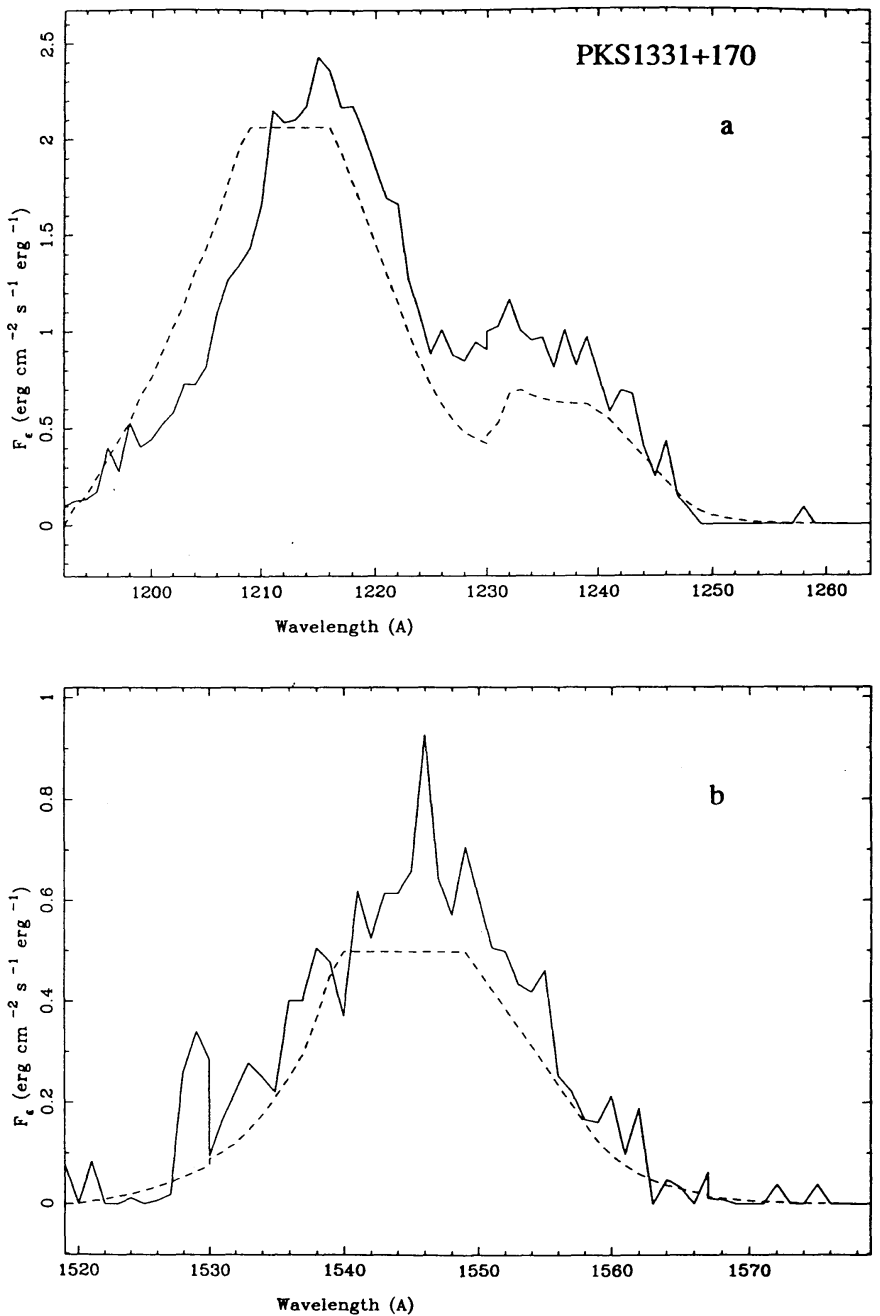

FIG. 10.-Comparison of observed (solid curve) and model (dashed curve) line profiles for the object PKS $1331+170$. (a) $\mathrm{Ly} \alpha / \mathrm{N} \vee \lambda 1240$ blend. (b) $\mathrm{C}$ IV $\lambda 1550$

tribution, possibly including, for example, a hot phase temperature which is constant throughout the BELR. However, the fits are significantly more sensitive to the fiducial temperature, $T_{0}$, since this affects the ionization parameter and pressure scale and hence the accessible line ratios. Equations (4) and (5) show that $T_{0}$ and $1 / \eta$ have the same effect on these quantities.

The objects with significantly differing hot phase temperature distribution, PG $1247+265$, PKS $1331+170$, and PKS 2212-299, all have Ly $\alpha$ lines which are broader than $\mathrm{C}$ IV 21550 . The best-fit parameters have hot phase temperature distributions which increase more rapidly with increasing velocity than do the majority. This has the effect of causing the ionization parameter to decrease, and the $\mathrm{Ly} \alpha / \mathrm{C}$ IV $\lambda 1550$ ratio to increase, with increasing velocity. The models also have a $N$ v $\lambda 1240$ line which is narrower than the $C$ IV $\lambda 1550$ line. The very extreme value of $\delta=d \log T_{\text {hot }} / d \log r$ required by the spectrum of $\mathrm{PG} 1247+265$, and the resulting poor fit, is perhaps the most obvious failure of our fitting program. A corollary to the extreme choice of hot phase temperature distribution for this object is the hot phase Thomson depth, which is much greater than unity. Such a depth will produce line profile changes via Compton broadening, and will almost certainly dominate the line broadening. Although we have not modeled these effects in this paper, we can predict that the lines would have broad $(\Delta \lambda / \lambda \geq 0.1)$ wings superposed on a narrow core of line photons which are emitted in the Thomson-thin parts of the BELR. Such profiles are clearly inconsistent with the observations.

\subsubsection{Covering Fraction Distribution}

The objects in our sample have covering fraction distributions which are similar, i.e., the value of $\epsilon$ in Table 6 between -6 and -8 , reflecting the basic similarity in the line profile shapes. The dependence of the profile shape on this quantity is illustrated in Figure 16, where the profile of $\mathrm{Ly} \alpha$ from the object Q1101-264 is shown superposed on several synthetic profiles with various values of $\epsilon$ between -7 and -9 . As discussed in $\S 2$, more negative values of $\epsilon$ cause the synthetic profile to be more rounded; the departures from the bolometric assumption cause the value necessary for this to occur to decrease from -3.5 to -6 .

\subsubsection{Accretion Efficiency}

The derived values of accretion efficiency, $\eta$, are clustered within a factor of 3 of each other for most of the objects. For
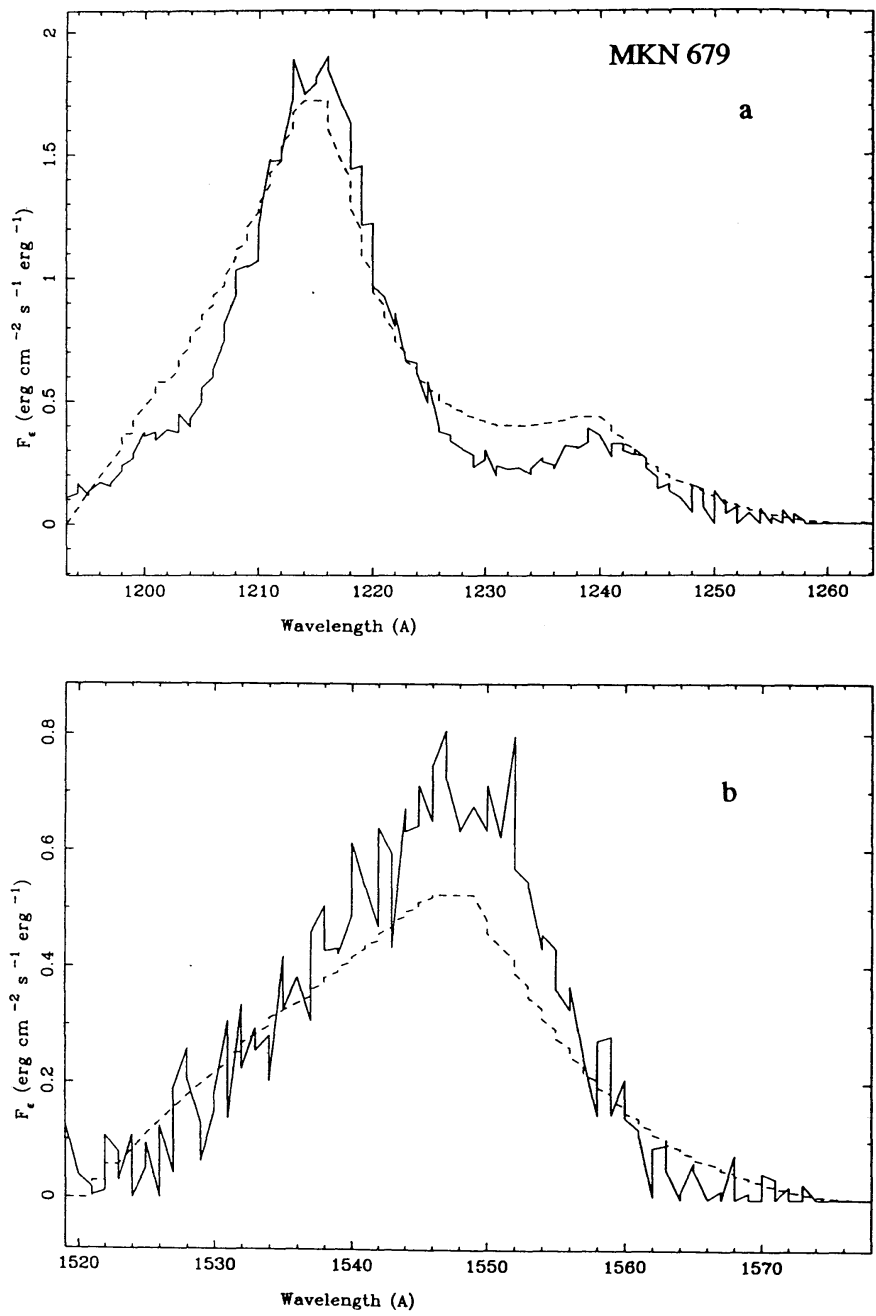

FIG. 11. - Comparison of observed (solid curve) and model (dashed curve) line profiles for the object Mrk 679. (a) $\mathrm{Ly} \alpha / \mathrm{N}$ v $\lambda 1240$ blend. (b) C IV $\lambda 1550$ 
Q0000-398, Q2204-408, PKS 2212-299 the derived values are lower than the average by $\sim 50$. This is a reflection of the fact that, for Q0000 - 398 and Q2204-408, the line strengths in require a significantly lower ionization parameter than do $\operatorname{lnI}$
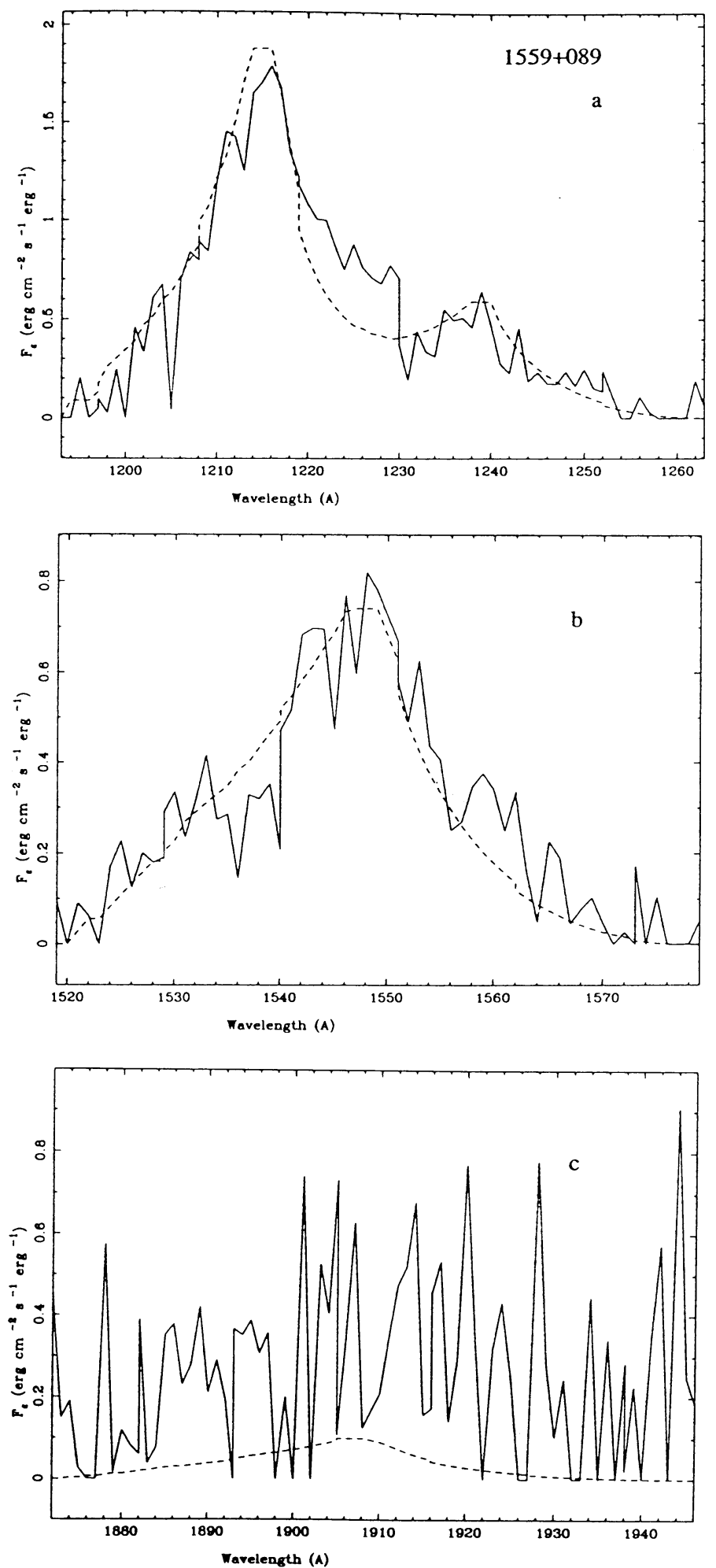

Fig. 12.-Comparison of observed (solid curve) and model (dashed curve) line profiles for the object $1559+089$. (a) $\mathrm{Ly} \alpha / \mathrm{N} \vee \lambda 1240$ blend. (b) $\mathrm{C}$ IV $\lambda 1550$. (c) C III] $\lambda 1909$ line. most of the other objects. For PKS 2212-299 this is also true, although the ionization parameter is not constant across the BELR owing to the differing widths of $\operatorname{Ly} \alpha$ and $C$ IV $\lambda 1549$. A much more extreme example of this is PG $1247+265$, for
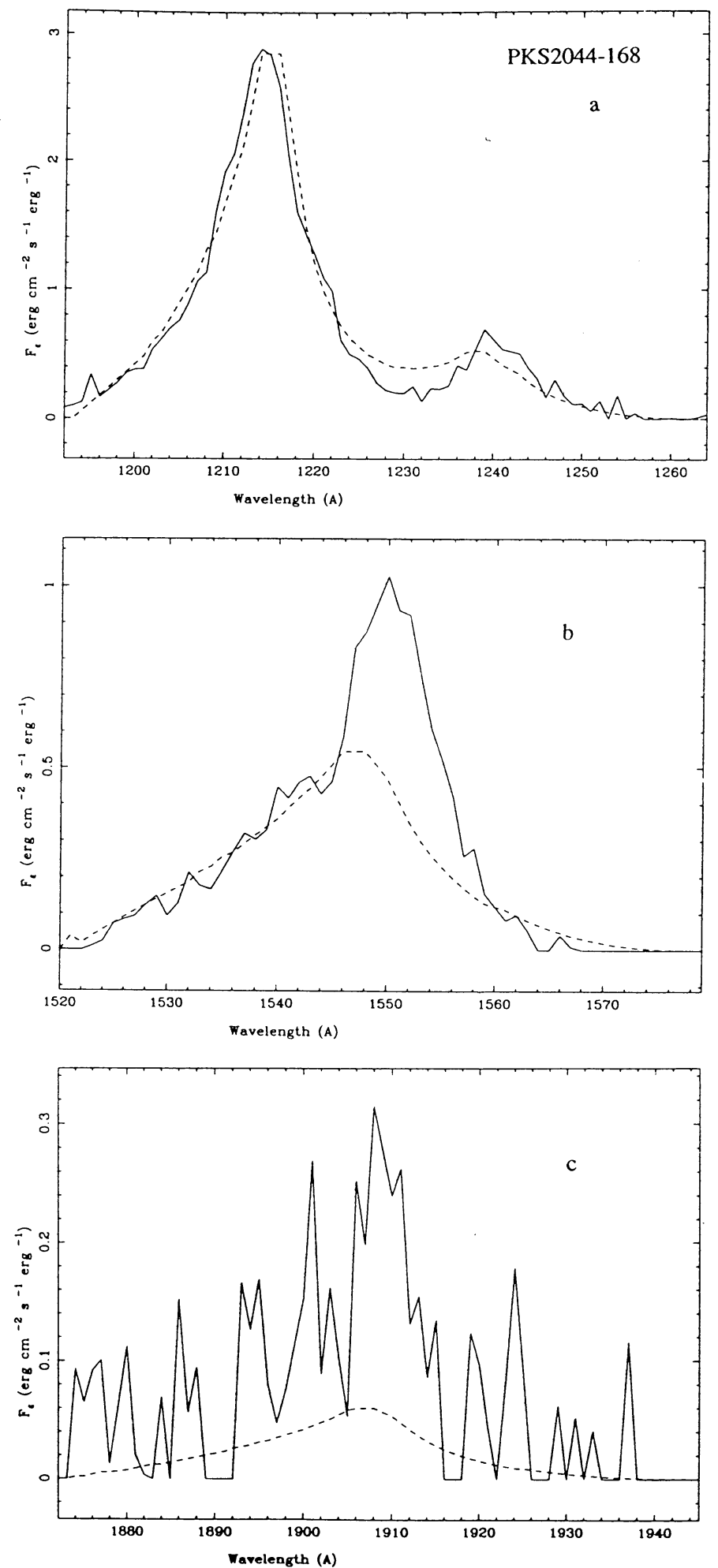

FIG. 13. - Comparison of observed (solid curve) and model (dashed curve) line profiles for the object PKS $2044-168$. (a) Ly $\alpha / \mathrm{N} \vee \lambda 1240$ blend. (b) $\mathrm{C}$ IV $\lambda 1550 .(c) \mathrm{C} \mathrm{III}] \lambda 1909$ line. 

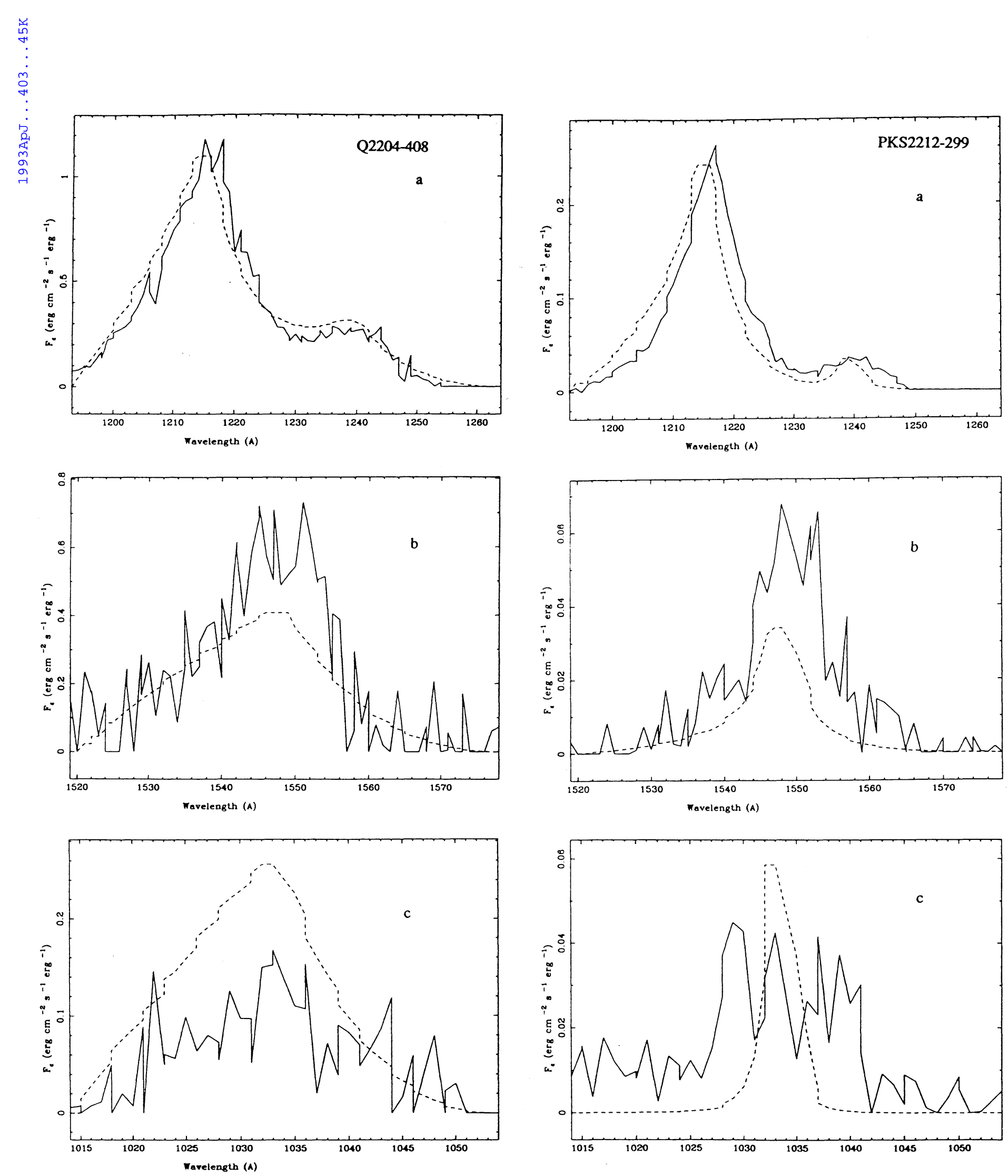

FIG. 14.-Comparison of observed (solid curve) and model (dashed curve) line profiles for the object Q2204-408. (a) Ly $\alpha / \mathrm{N} v \lambda 1240$ blend. (b) $\mathrm{C}$ IV $\lambda 1550 .(c) O$ vi 21034 line.

Fig. 15.-Comparison of observed (solid curve) and model (dashed curve) line profiles for the object PKS 2212-299. (a) Ly $\alpha / \mathrm{N}$ v $\lambda 1240$ blend. (b) C IV 21550. (c) O vi $\lambda 1034$ line. (d) C III] $\lambda 1909$ line. 


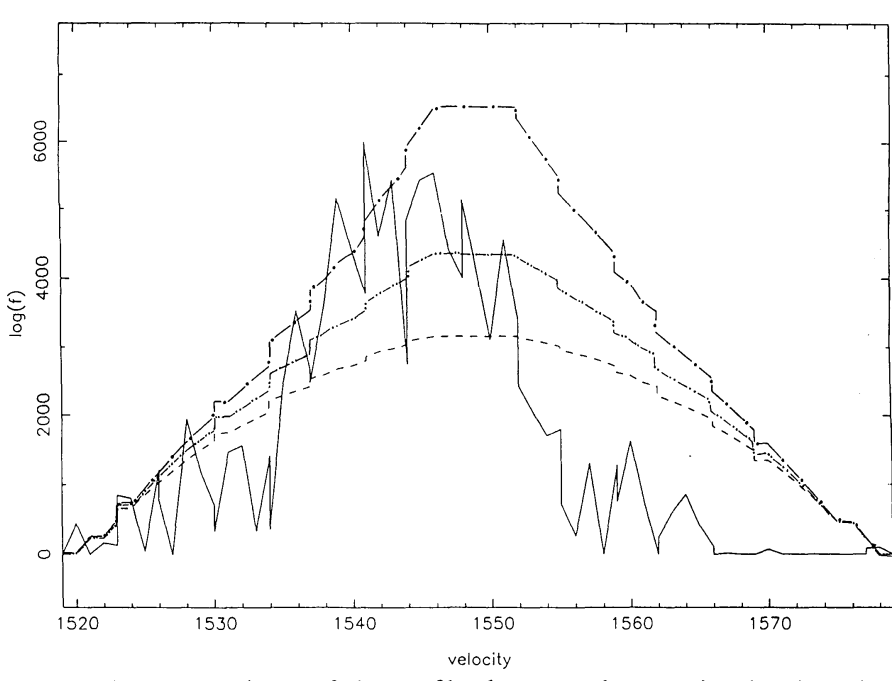

FIG. 16.-Dependence of the profile shape on the covering fraction distribution. The profile of $L y \alpha$ from the object Q1101-264 is shown superposed on several synthetic profiles with various values of $\epsilon$ between -7 and -9 .

which the derived accretion efficiency is the smallest in our sample.

\subsubsection{Redshift}

One consequence of infall dynamics together with anisotropically radiating clouds is that the centroids, and to a lesser extent the peaks, of model profiles are blueshifted relative to the rest velocity of the quasar. This is qualitatively consistent with the observed discrepancy between broad- and narrow-line redshifts from many objects. We have tested for this effect by repeating our fitting procedure with various choices of redshift between the BELR and the $\mathrm{O}$ I values given in Table 1 . We find that for the majority of objects better fits are obtained with the BELR redshift than with the $O$ I redshift. The only counterexample is Q0000 - 398, for which we obtain a better fit with a redshift 2.835 which is between the BELR and $\mathrm{O}_{\mathrm{I}}$ values.

\section{DISCUSSION}

The results of our model fits are best summarized by enumerating what we consider to be their successes and their failings. On the success side is the fact that, in an average sense, the observed profiles from many of our sample of objects are fitted by a simple dynamical model, that of freely falling clouds, together with an accurate description of the emission properties of the BELR clouds. By this we mean that throughout the range of wavelengths where the observed line exceeds the uncertainties in the continuum placement, the models for the strongest lines ( $\mathrm{Ly} \alpha, \mathrm{N} v$, and $\mathrm{C}$ IV) agree with the observed profile to within the uncertainties for most lines. This includes the three major observables associated with the lines: the relative strengths of the various lines from each object, the asymmetries of the lines, and the shape of each "wing" of a given line. We also reiterate that these fits were achieved using freeparameter values which are not greatly different from the average over the sample; we did not have to search exhaustively to find the fits displayed in the previous section. We also note the qualified success of one particular choice of cloud radiation pattern in fitting all the lines from all the objects. This occurs in spite of the fact that C IV $\lambda 1549$ generally appears to be more asymmetric than Ly $\alpha$, and is largely due to the effect of $\mathrm{N} \vee \lambda 1240$ in masking the asymmetry of Ly $\alpha$.
Furthermore, we find a relatively small dispersion in the values of the best-fit parameters. For example, the accretion efficiencies and covering fractions of most of the objects span a range of less than a factor of 3 , while the continuum luminosities span a factor of 20 . The covering fraction distributions are also all very similar. In addition, we are convinced that, for most lines, improved fits could be found if the parameter values were refined slightly or if a different choice of cloud radiation pattern were employed. These conclusions are affected by the statistical quality of the data we employ; many of the spectra contain hints that, if the uncertainties were smaller, more significant discrepancies would arise between data and models.

The formal success of these fits, and the similarity of the derived parameters for nearly all the objects in our sample, would at first glance suggest that these models might actually describe quasar BELRs. In only one case out of 12 (the Ly $\alpha /$ $\mathrm{N} v \lambda 1240$ blend in PG $1247+265$ ) were we unable to find parameters giving and adequate fit (or even a self-consistent fit, owing to the large implied Thomson depth of the hot phase). However, two problems undercut this formal success: when data for C III] $\lambda 1909$ are also available, more often than not no acceptable fit can be found for all the lines; and the derived parameters are in conflict with those estimated on other grounds.

The central mass required to produce the observed velocities by free fall can be estimated to order of magnitude once the ionization parameter and cloud pressure are known. Because these were first established (at the order-of magnitude level for single-zone models) a decade ago, it has long been known that central masses of at least $10^{10} L_{46}^{1 / 2} M_{\odot}$ would be necessary for the free-fall model to succeed. Our more detailed fits find even larger central masses: $\left(M / M_{\odot}\right) / L_{46}^{1 / 2}$ ranges from $5 \times 10^{10}$ to $3 \times 10^{12}$. Although quasars of this luminosity are rare enough so that the nearest black hole is probably far enough away that masses $\sim 10^{10} M_{\odot}$ could not yet be ruled out, the effect on stellar orbits of a $10^{12} M_{\odot}$ remnant would extend over an entire host galaxy, and we would likely have found examples by now if they existed. Such large remnant masses would also imply that the bulk of the accretion occurred with low efficiency.

In a gravitational free-fall model, large central mass has the corollary of large distance from the central mass to the emission-line region, and hence low pressure in the emissionline clouds. Because we have no data on lines such as [O III] $\lambda 5007, \mathrm{He} \mathrm{I} \lambda 5876$, and $\mathrm{H} \alpha$ or $\mathrm{H} \beta$, our model fits were rather insensitive to the strongest lower bounds on the pressure in the BELR. If data had been available for these lines, we think it quite likely that the fits we obtained would no longer be acceptable. For this reason, we think that these detailed profile fits to the rest-frame ultraviolet lines have come close to ruling out the simplest model for the quasar BELR - spherically symmetric gravitational free fall.

Our results are also dependent on the assumptions we have made about the ionizing radiation spectrum incident on the broad-line clouds. We have adopted a broken-power-law spectrum with relatively strong hard $\mathrm{X}$-rays and with no large "blue bump" extending into the extreme ultraviolet and soft $\mathrm{X}$-ray spectral regions. This spectrum is more successful at reproducing the total line strengths for a single-zone model than are spectra with a strong soft excess, in spite of the fact that such spectra have been observed from a large number of active galaxies (e.g., Wilkes \& Elvis 1987). This may be a reflection of the shortcomings of our cloud-modeling procedure, or 
it may be due to geometrical effects which prevent the BELR clouds from seeing the same ionizing spectrum that we observe.

Other dynamical scenarios might be more successful in accounting for the observed line strengths and profiles. These include outflows driven by radiation pressure, particle winds, thermal winds, or turbulent or predominantly Keplerian flows.

- Although these deserve as careful attention as we have devoted to free fall, they suffer from uncertainty concerning the mechanism for producing the line asymmetries. All require the asym- metry to be produced by absorption by material outside the clouds, and the origin and distribution of such material are highly uncertain.

We thank Harvey Tananbaum and the High Energy Astrophysics group at CfA for support and encouragement during the completion of this project, and Hagai Netzer and Suzy Collin-Souffrin for many constructive comments and criticisms.
Adams, G. 1985, A\&AS, 61, 225

Baldwin, J. 1975, ApJ, 201, 26

. 1977, ApJ, 214, 679

Baldwin, J., \& Netzer, H. 1978, ApJ, 226, 1

Blair, M., \& Gilmore, G. 1982, PASP, 94, 742

Capriotti, E., Foltz, C., \& Byard, P. 1979, ApJ, 230, 681

Clavel, J., et al. 1991, ApJ, 366, 64

Collin-Souffrin, S., \& Dumont, A. 1986, A\&A, 166, 13

Davidson, K., \& Netzer, H. 1979, Rev. Mod. Phys., 51, 715

Espey, B., Carswell, R., Bailey, J., Smith, M., \& Ward, M. 1989, ApJ, 266, 110

Ferland, G., \& Mushotzky, R. F. 1982, ApJ, 262, 564

Ferland, G., \& Netzer, H. 1983, ApJ, 264, 105

Ferland, G., Netzer, H., \& Shields, G. 1979, ApJ, 232, 382

Ferland, G. J., \& Persson, E. 1989, ApJ, 347, 656

Gaskell, M. 1982, ApJ, 263, 79

Hewitt, A., \& Burbidge, G. 1989, ApJ, 69, 1

Kallman, T. R., \& Krolik, J. H. 1986, ApJ, 308, 805

Kallman, T. R., \& Lepp, S. 1987, ApJ, 321, 907

Kallman, T. R., \& Mushotzky, R. F. 1986, ApJ, 292, 49

Krolik, J. H. 1988, ApJ, 325, 148

Krolik, J. H., Horne, K., Kallman, T. R., Kriss, J., \& Malkan, M. 1991, ApJ, 371,541

Krolik, J. H., \& Kallman, T. R. 1988, ApJ, 324, 714

Krolik, J. H., \& London, R. 1983, ApJ, 267, 18

Krolik, J. H., McKee, C. F. \& Tarter, C. B. 1981, ApJ, 249, 422

Kwan, J., \& Carroll, B. 1982, ApJ, 261, 25

Kwan, J., \& Krolik, J. H. 1981, ApJ, 250, 478

Mathews, W. 1974, ApJ, 189, 23

. 1986, ApJ, 305, 187

\section{REFERENCES}

Mathews, W., \& Ferland, G. 1987, ApJ, 323, 456

Mobasher, B. \& Raine, D. J. 1990, MNRAS, 244, 652

Netzer, H. 1977, MNRAS, 181, 89P 1987, MNRAS, 225, 55

Osmer, P. S., \& Smith, M. G. 1980, ApJS, 42, 333

Peterson, G. J., et al. 1991, ApJ, 368, 119

Pounds, K. A., Nandra, K., Stewart, G. C., George, I. M., \& Fabian, A. C. 1990 Nature, 344, 132

Press, W., Flannery, W., Teukolsky, S., \& Vetterling, W. 1986, Numerical Recipes (Cambridge: Cambridge Univ. Press)

Puetter, R. C., \& Hubbard, E. N. 1983, ApJ, 273, 36

Rees, M., Netzer, H., \& Ferland, G. 1989, ApJ, 347, 640

Schmidt, M., \& Green, R. F. 1983, ApJ, 269, 352

Shields, G., \& McKee, C. 1981, ApJ, 246, L57

Shlosman, I., Vitello, P. A., \& Shaviv, G. 1985, ApJ, 294, 96

Trimble, V. 1975, Rev. Mod. Phys., 47, 877

Weisheit, J., Shields, G., \& Tarter, C. B. 1982, ApJ, 245, 406

Weymann, R. J., Scott, J. S., Schiano, A. V. R., \& Christiansen, W. A. 1982 ApJ, 262, 497

Wilkes, B. J. 1984, MNRAS, 207, 73

1986, MNRAS, 218, 331

Wilkes, B. J., \& Carswell, R. F. 1982, MNRAS, 201, 645

Wilkes, B. J., \& Elvis, M. 1987, ApJ, 323, 243

Wilkes, B. J., Tananbaum, H., Avni, Y., Oey, M. S., \& Worrall, D. M. 1990, in Imaging X-Ray Astronomy: A Decade of Einstein Observatory Achievements, ed. M. Elvis (Cambridge: Cambridge Univ. Press), 327

Wilkes, B. J., Tananbaum, H., Worrall, D. M., Avni, Y., Oey, M. S., \& Flanagan, J. 1991, in preparation

Wills, B., Netzer, H., \& Wills, D. 1985, ApJ, 288, 94 